\title{
HALL-LITTLEWOOD FUNCTIONS, PLANE PARTITIONS, AND THE ROGERS-RAMANUJAN IDENTITIES
}

\author{
JOHN R. STEMBRIDGE
}

\begin{abstract}
We apply the theory of Hall-Littlewood functions to prove several multiple basic hypergeometric series identities, including some previously known generalizations of the Rogers-Ramanujan identities due to $G$. E. Andrews and D. M. Bressoud. The techniques involve the adaptation of a method due to I. G. Macdonald for calculating partial fraction expansions of certain types of symmetric formal power series. Macdonald originally used this method to prove a pair of generating function identities for plane partitions conjectured by MacMahon and Bender-Knuth. We show that this method can also be used to prove another pair of plane partition identities recently obtained by $R$. A. Proctor.
\end{abstract}

\section{INTRODUCTION}

Many identities from the theory of symmetric functions can be viewed as generalizations of standard results from the theory of partitions and/or basic hypergeometric series. See Chapter I of $[\mathrm{M}]$, for example. The sense of genralization typically derives from the fact that when the variables $x_{1}, x_{2}, \ldots$ in a symmetric function identity are specialized to be powers of a single variable $q$, one usually obtains combinatorial information about partitions (linear or plane), or else a well-known fact about basic hypergeometric series, i.e., $q$ series. However, most of the results that have been obtained in this way do not extend very deeply into the theory of $q$-series.

The main purpose of this paper is to present methods for deriving some nontrivial $q$-series identities via the theory of Hall-Littlewood symmetric functions. For example, we will obtain the Rogers-Ramanujan identities

$$
\begin{aligned}
& \sum_{r=0}^{\infty} \frac{q^{r^{2}}}{(1-q)\left(1-q^{2}\right) \cdots\left(1-q^{r}\right)}=\prod_{n=1}^{\infty}\left(1-q^{5 n-1}\right)^{-1}\left(1-q^{5 n-4}\right)^{-1}, \\
& \sum_{r=0}^{\infty} \frac{q^{r(r+1)}}{(1-q)\left(1-q^{2}\right) \cdots\left(1-q^{r}\right)}=\prod_{n=1}^{\infty}\left(1-q^{5 n-2}\right)^{-1}\left(1-q^{5 n-3}\right)^{-1}
\end{aligned}
$$

as a consequence of these methods.

Received by the editors January 23, 1989.

1980 Mathematics Subject Classification (1985 Revision). Primary 05A19, 05A17, 05A30, 11 P68.

Partially supported by an NSF Postdoctoral Research Fellowship. The author was also supported by the Institute for Mathematics and its Applications with funds provided by the NSF. 
The most crucial part of the method we present was suggested by Macdonald's derivation of a partial fraction expansion involving Hall-Littlewood functions (see Example III.5.5 in [M]). Macdonald [M] used this expansion to prove a pair of plane partition conjectures due to MacMahon (Example I.5.17), and Bender and Knuth (Example I.5.19), and to calculate the Hecke series for the group of symplectic similitudes over a local field $(\S \mathrm{V} .5)$. By specializing the variables in Macdonald's expansion, one obtains an interesting pair of apparently new multiple $q$-series identities (see Corollary 1.5(a) and (b) below), but not, unfortunately, the Rogers-Ramanujan identities. Nevertheless, in $\S 1$, we will derive another Hall-Littlewood identity using Macdonald's method that will imply not only the Rogers-Ramanujan identities, but also a pair of generalizations due originally to Andrews [A2] (see Corollary 1.5(c) and (d) below).

In view of the unusual nature of this proof of the Rogers-Ramanujan identities, it is natural to inquire as to whether the theory of symmetric functions in general, or Hall-Littlewood functions in particular, is unavoidably required by these methods. In fact, they are not; in $\S 2$ we give a redevelopment of the main steps in $\S 1$ that is entirely elementary. (Although it would be foolish to claim we have contradicted Hardy's assertion that none of the proofs of the RogersRamanujan identities can be called both "simple" and "straightforward.")

The techniques we present in $\S 2$ show that Macdonald's method can be applied directly to certain types of $q$-series, rather than symmetric functions. This observation suggests the possibility of deriving further identities of the RogersRamanujan type by working directly with $q$-series. This possibility is realized in $\S 3$, where we use Macdonald's method to prove even more general results, culminating in 16 families of multiple $q$-series identities of the Rogers-Ramanujan type. Some of these identities can be found in the vast catalogue of multiple $q$-series identities due to Andrews [A2, A3] and Bressoud [B1, B2] but others appear to be new.

There have also been many combinatorial generalizations of the RogersRamanujan identities, beginning with Gordon [G1], and later with Andrews [A1, A3] and Bressoud [B1-B3]. However, we have made no attempt to recast the $q$-series identities we prove here in combinatorial terms.

Finally, in $\S 4$ we return to one of the original purposes of Macdonald's partial fraction method: the enumeration of plane partitions. There are a number of known or conjectured generating function formulas for sets of plane partitions belonging to various types of symmetry classes (see [St] for a survey). Two of these are the pair mentioned earlier; these were proved not only by Macdonald, but also independently by Andrews [A7, A8], Gordon [G2], and Proctor [P1]. Recently, two more plane partition generating function formulas were found by Proctor [P2]; we will show that Macdonald's method can be adapted to prove this new pair as well.

Among the further applications of Macdonald's method, we remark that it has been recently used by Goulden to enumerate Young tableaux of bounded width [Go]. 
Acknowledgements. The research for this paper began in March of 1988 while I was visiting the Institute for Mathematics and its Applications (IMA). In the span of a few days, I had a succession of fortuitous, coincidental discussions with several other visitors that allowed me to stumble upon the strange connections between Hall-Littlewood functions and $q$-series that are the subject of this paper. I would like to thank the IMA (and the organizers) for providing the opportunity for this good fortune.

Notation and background. For any nonnegative integer $n$ and indeterminates $z, q$ we will use the abbreviations

$$
\begin{aligned}
& (z)_{n}=(1-z)(1-q z) \cdots\left(1-q^{n-1} z\right), \\
& (z)_{\infty}=(1-z)(1-q z)\left(1-q^{2} z\right) \cdots,
\end{aligned}
$$

with the dependence on $q$ implicitly understood. In circumstances where the $q$ dependence needs to be displayed explictly, we will write $(z ; q)_{n}$ and $(z ; q)_{\infty}$. For future reference, let us record here Jacobi's Triple Product Identity [A9, $(7.1)]$ :

$$
(q)_{\infty}(a)_{\infty}(q / a)_{\infty}=\sum_{r=-\infty}^{\infty}(-1)^{r} a^{r} q^{\left(\begin{array}{l}
r \\
2
\end{array}\right)},
$$

and the $q$-Binomial Identity [A9, (2.9)]:

$$
\frac{(a z)_{\infty}}{(z)_{\infty}}=\sum_{r=0}^{\infty} z^{r} \frac{(a)_{r}}{(q)_{r}}
$$

Note that the terms of the two-sided series $(0.1)$ can be combined and rearranged into the following equivalent pair of one-sided series:

$$
\begin{aligned}
& (q)_{\infty}(a)_{\infty}(q / a)_{\infty}=1+\sum_{r=1}^{\infty}(-1)^{r} a^{r} q^{\left(\begin{array}{c}
r \\
2
\end{array}\right)}\left(1+q^{r} / a^{2 r}\right), \\
& (q)_{\infty}(a)_{\infty}(q / a)_{\infty}=\sum_{r=0}^{\infty}(-1)^{r} a^{r} q^{\left(\begin{array}{c}
r \\
2
\end{array}\right)}\left(1-q^{2 r+1} / a^{2 r+1}\right) .
\end{aligned}
$$

Also, if we replace $q$ with $q^{-1}$ and $z$ with $-z / q$, the special case $a=q^{n}$ of (0.2) becomes the following well-known $q$-analogue of the Binomial Theorem:

$$
(-z ; q)_{n}=\sum_{r=0}^{n} z^{r} q^{\left(\begin{array}{l}
r \\
2
\end{array}\right)}\left[\begin{array}{l}
n \\
r
\end{array}\right]
$$

where $\left[\begin{array}{l}n \\ r\end{array}\right]=(q)_{n} /(q)_{r}(q)_{n-r}$ denotes the $q$-binomial coefficient.

A partition of $r$ is a weakly decreasing sequence $\lambda=\left(\lambda_{1}, \lambda_{2}, \ldots\right)$ of nonnegative integers such that $|\lambda|=\sum \lambda_{i}=r$. The number of nonzero terms of $\lambda$ is called the length and denoted by $\ell(\lambda)$. The conjugate $\lambda^{\prime}$ is the partition whose $i$ th term $\lambda_{i}^{\prime}$ is defined to be the number of terms $\geq i$ in $\lambda$. In most cases, we 
will follow the partition notation of [M], although one important exception will be the parameter

$$
n(\lambda):=\sum_{i}\left(\begin{array}{c}
\lambda_{i} \\
2
\end{array}\right)
$$

This quantity would be $n\left(\lambda^{\prime}\right)$ in the notation of $[\mathrm{M}]$.

It will be convenient to extend the notation $(z)_{n}$ to partitions $\lambda$ by defining

$$
(z)_{\lambda}=(z)_{\lambda_{1}-\lambda_{2}}(z)_{\lambda_{2}-\lambda_{3}} \cdots,
$$

and to extend the $q$-binomial notation by defining

$$
\left[\begin{array}{l}
n \\
\lambda
\end{array}\right]=\frac{(q)_{n}}{(q)_{n-\lambda_{1}}(q)_{\lambda}}
$$

with the convention that $\left[\begin{array}{l}n \\ \lambda\end{array}\right]=0$ if $\lambda_{1}>n$.

Finally, if $x=\left(x_{1}, \ldots, x_{n}\right)$ is an $n$-tuple of variables and $\alpha \in \mathbf{Z}^{n}$ (or even $\mathbf{R}^{n}$ ), we will use the notation $x^{\alpha}$ as an abbreviation for $x_{1}^{\alpha_{1}} \cdots x_{n}^{\alpha_{n}}$.

\section{BASIC TECHNIQUES}

For each partition $\lambda=\left(\lambda_{1}, \ldots, \lambda_{n}\right)$ of length at most $n$, let $P_{\lambda}(x)=$ $P_{\lambda}(x ; q)$ denote the Hall-Littlewood function indexed by $\lambda$ in the variables $x=\left(x_{1}, \ldots, x_{n}\right)$; i.e.,

$$
P_{\lambda}(x ; q)=\sum_{w \in S_{n} / \lambda} w\left[x^{\lambda} \prod_{\lambda_{i}>\lambda_{j}} \frac{x_{i}-q x_{j}}{x_{i}-x_{j}}\right]
$$

where $S_{n} / \lambda$ denotes a set of left coset representatives for the subgroup of the symmetric group $S_{n}$ consisting of those permutations of $\left(x_{1}, \ldots, x_{n}\right)$ that fix $x^{\lambda}[\mathrm{M}, \mathrm{III}]$.

Let $\mu_{1}>\mu_{2}>\cdots>\mu_{l} \geq 0$ denote the distinct integers occurring among $\left(\lambda_{1}, \ldots, \lambda_{n}\right)$, and let $m_{i}$ denote the multiplicity of $\mu_{i}$ in $\lambda$. The $l$-tuple $(m)=\left(m_{1}, \ldots, m_{l}\right)$ is thus a composition of $n$. The distinct permutations of $x^{\lambda}$ may be indexed by (ordered) partitions of the indeterminate-set $x$ into blocks of sizes $m_{1}, \ldots, m_{l}$, or equivalently, by functions $f: x \rightarrow\{1,2, \ldots, l\}$ with $\left|f^{-1}(i)\right|=m_{i}$. We will refer to such a map $f$ as a function of type $(m)$. Using $f_{i}$ as an abbreviation for the product of the variables in $f^{-1}(i)$, the definition (1.1) may be rewritten in the form

$$
P_{\lambda}(x ; q)=\sum_{f} f_{1}^{\mu_{1}} \ldots f_{l}^{\mu_{l}} \pi_{f}, \quad \pi_{f}=\prod_{f\left(x_{i}\right)<f\left(x_{j}\right)} \frac{x_{i}-q x_{j}}{x_{i}-x_{j}},
$$

summed over functions of type $(m)$.

It is well known and not difficult to show that the Hall-Littlewood functions are symmetric polynomial functions of the variables $x_{1}, \ldots, x_{n}$. In fact, they form a basis of $R\left[x_{1}, \ldots, x_{n}\right]^{S_{n}}$ as a module over the coefficient ring $R=\mathbf{Z}[q]$ 
(see [M, III]). An alternative definition which makes the symmetry of $P_{\lambda}$ more transparent is the the following:

$$
P_{\lambda}(x ; q)=\frac{1}{v_{\lambda}(q)} \sum_{w \in S_{n}} w\left[x^{\lambda} \prod_{i<j} \frac{x_{i}-q x_{j}}{x_{i}-x_{j}}\right],
$$

where $v_{\lambda}(q)=\prod_{i=1}^{l}(q)_{m_{i}} /(1-q)^{m_{i}}$ [M, III.(2.1)]. From this definition it is also clear that $P_{\lambda}(x ; 0)$ is the Schur function $s_{\lambda}(x)$.

The principal source from which we intend to derive $q$-series identities develops from the following pair of Hall-Littlewood function expansions:

$$
\begin{gathered}
\Phi(x ; q)=\sum_{\ell(\lambda) \leq n} P_{\lambda}(x ; q), \\
\Psi(x ; q)=\sum_{\ell(\lambda) \leq n} P_{2 \lambda}(x ; q),
\end{gathered}
$$

where $\Phi(x ; q)$ and $\Psi(x ; q)$ are the $n$-variate symmetric formal power series defined by

$$
\begin{aligned}
& \Phi(x ; q)=\prod_{i} \frac{1}{1-x_{i}} \prod_{i<j} \frac{1-q x_{i} x_{j}}{1-x_{i} x_{j}}, \\
& \Psi(x ; q)=\prod_{i} \frac{1}{1-x_{i}^{2}} \prod_{i<j} \frac{1-q x_{i} x_{j}}{1-x_{i} x_{j}} .
\end{aligned}
$$

Proofs may be found in Examples III.5.1, 2 of [M].

Starting with identity (1.4), Macdonald developed a generalization in which sums analogous to (1.4) were restricted to those partitions $\lambda$ whose diagrams fit inside an $n \times k$ rectangle (i.e., $\lambda_{1} \leq k$ and $\ell(\lambda) \leq n$; see Example III.5.5, op. cit.). This generalization led to Macdonald's proof of the plane partition conjectures and Hecke series calculations mentioned in the introduction.

To describe this result, let us define $\theta=(1, \ldots, 1) \in \mathbf{Z}^{n}$, and use $\mathbf{Z}_{2}^{n}$ to denote the elementary abelian group of order $2^{n}$ acting on $\mathbf{Z}^{n}$ via sign changes and on the variables $x_{i}$ by inversions. Thus, for a given element $s \in \mathbf{Z}_{2}^{n}, s \theta$ is obtained by negating certain entries of $\theta$ and $s x$ is obtained by replacing certain variables $x_{i}$ with $1 / x_{i}$. By convention, $P_{\lambda}(x ; q)=0$ for $\ell(\lambda)>n$, so the constraint $\ell(\lambda) \leq n$ need not be explictly imposed in what follows.

Theorem 1.1 (Macdonald). We have

$$
\sum_{k \geq 0} u^{k} \sum_{\lambda_{1} \leq k} P_{\lambda}(x ; q)=\sum_{s \in \mathbf{Z}_{2}^{n}} \Phi(s x ; q) \frac{1}{1-u x^{(\theta-s \theta) / 2}} .
$$

The following result is an analogous generalization of (1.5). The proof we give is quite similar to Macdonald's proof of Theorem 1.1. 
Theorem 1.2. We have

Proof. Define

$$
\sum_{k>0} u^{k} \sum_{\lambda_{1} \leq k} P_{2 \lambda}(x ; q)=\sum_{s \in \mathbf{Z}_{2}^{n}} \Psi(s x ; q) \frac{1}{1-u x^{\theta-s \theta}} .
$$

$$
\psi(u)=\sum_{k \geq 0} u^{k} \sum_{\lambda_{1} \leq k} P_{2 \lambda}(x ; q)=\frac{1}{1-u} \sum_{\lambda} u^{\lambda_{1}} P_{2 \lambda}(x ; q),
$$

and observe that (1.5) implies

$$
\left.\psi(u)(1-u)\right|_{u=1}=\Psi(x ; q) .
$$

Using (1.2), we may rewrite the definition of $\psi(u)$ in the form

$$
\psi(u)=\frac{1}{1-u} \sum_{\mu, f} f_{1}^{2 \mu_{1}} \cdots f_{l}^{2 \mu_{l}} u^{\mu_{1}} \pi_{f}
$$

summed over all partitions $\mu=\left(\mu_{1}>\mu_{2}>\cdots>\mu_{l} \geq 0\right)$ and all surjective functions $f: x \rightarrow\{1, \ldots, l\}$ with $l=1,2,3, \ldots$. thus

If we set $\nu_{i}=\mu_{i+1}(i<l)$ and $\nu_{l}=\mu_{l}$, then $\nu_{1}, \ldots, \nu_{l-1}>0, \nu_{l} \geq 0$ and

$$
\sum_{\mu} f_{1}^{2 \mu_{1}} \cdots f_{l}^{2 \mu_{l}} u^{\mu_{1}}=\sum_{\nu} F_{1}^{2 \nu_{1}} \cdots F_{l}^{2 \nu_{l}} u^{\nu_{1}+\cdots+\nu_{l}}=\left\langle u F_{1}^{2}\right\rangle \cdots\left\langle u F_{l-1}^{2}\right\rangle \frac{1}{1-u x^{2 \theta}},
$$

where $\langle z\rangle:=z /(1-z)$ and $F_{i}:=f_{1} f_{2} \cdots f_{i}$. We therefore have

$$
\psi(u)=\frac{1}{1-u} \sum_{f}\left\langle u F_{1}^{2}\right\rangle \cdots\left\langle u F_{l-1}^{2}\right\rangle \frac{\pi_{f}}{1-u x^{2 \theta}} .
$$

This expansion shows that $\psi(u)$ is a rational function of $u$ with simple poles occurring at $u=x^{-2 \alpha}\left(\alpha \in\{0,1\}^{n}\right)$. Since $\psi(u)$ vanishes at infinity, there must be a partial fraction decomposition of the form

$$
\psi(u)=\sum_{\alpha \in\{0,1\}^{n}} \frac{c_{\alpha}}{1-u x^{2 \alpha}},
$$

where $c_{\alpha}$ is the limit of $\psi(u)\left(1-u x^{2 \alpha}\right)$ as $u \rightarrow x^{-2 \alpha}$.

From (1.6), we see that $c_{0}=\Psi(x ; q)$, and therefore (cf. (1.7)),

$$
\Psi(x ; q)=\sum_{f}\left\langle F_{1}^{2}\right\rangle \cdots\left\langle F_{l-1}^{2}\right\rangle \frac{\pi_{f}}{1-x^{2 \theta}} .
$$

Note that $\pi_{f}\left(x^{-1}\right)=\pi_{\widehat{f}}(x)$, where $\widehat{f}\left(x_{i}\right)=l+1-f\left(x_{i}\right)$. Hence,

$$
\begin{aligned}
\Psi\left(x^{-1} ; q\right) & =\sum_{f}\left\langle F_{1}^{-2}\right\rangle \cdots\left\langle F_{l-1}^{-2}\right\rangle \frac{\pi_{f}\left(x^{-1}\right)}{1-x^{-2 \theta}} \\
& =\frac{1}{1-x^{-2 \theta}} \sum_{\widehat{f}}\left\langle x^{-2 \theta} \widehat{F}_{1}^{2}\right\rangle \cdots\left\langle x^{-2 \theta} \widehat{F}_{l-1}^{2}\right\rangle \pi_{\widehat{f}},
\end{aligned}
$$


and therefore (cf. (1.7)), $c_{\theta}=\Psi\left(x^{-1} ; q\right)$. More generally, a typical summand of (1.7) will contribute to $c_{\alpha}$ iff $x^{\alpha}$ occurs among the partial products $F_{i}(0 \leq$ $i \leq l)$. Restricting our attention to the maps $f$ with this property, we find $c_{\alpha}=\sum_{f}\left\langle x^{-2 \alpha} F_{1}^{2}\right\rangle \cdots\left\langle x^{-2 \alpha} F_{j-1}^{2}\right\rangle \frac{1}{1-x^{-2 \alpha}}\left\langle x^{-2 \alpha} F_{j+1}^{2}\right\rangle \cdots\left\langle x^{-2 \alpha} F_{l-1}^{2}\right\rangle \frac{\pi_{f}}{1-x^{2(\theta-\alpha)}}$, where $j$ denotes the index (depending on $f$ ) for which $F_{j}=x^{\alpha}$. Let $s$ denote the member of $\mathbf{Z}_{2}^{n}$ for which $2 \alpha=\theta-s \theta$, and let $x_{+}, x_{-}$denote the sets of noninverted and inverted variables in $s x$, respectively. Using the fact that

$$
\pi_{f}=\left.\left.\pi_{f}\right|_{x_{-}} \cdot \pi_{f}\right|_{x_{+}} \cdot \prod_{x_{i} \in x_{-}, x_{j} \in x_{+}} \frac{1-q x_{j} x_{i}^{-1}}{1-x_{j} x_{i}^{-1}}
$$

an application of (1.8) and (1.9) therefore yields

$$
c_{\alpha}=\Psi\left(x_{-}^{-1} ; q\right) \Psi\left(x_{+} ; q\right) \prod_{x_{i} \in x_{-}, x_{j} \in x_{+}} \frac{1-q x_{j} x_{i}^{-1}}{1-x_{j} x_{i}^{-1}}=\Psi(s x ; q) .
$$

This determines the partial fraction expansion of $\psi(u)$, and so the result follows.

We remark that there are other Hall-Littlewood function identities similar to (1.4) and (1.5) (see Examples III.5.3,4 of [M]) to which the above techniques may be applied. However, in these other cases there arise complications which render doubtful the existence of expansions as explicit as those of Theorems 1.1 and 1.2 .

We now consider the effect of the substitution $x_{i}=z q^{i-1}(1 \leq i \leq n)$ on the identities of Theorems 1.1 and 1.2. Using the notation introduced in $\S 0$, we claim that the following $q$-series identities are the result.

Theorem 1.3.

(a) $\sum_{\ell(\lambda) \leq k} z^{|\lambda|} q^{n(\lambda)}\left[\begin{array}{l}n \\ \lambda\end{array}\right]=(-z)_{n} \sum_{r=0}^{n}(-1)^{r} z^{(k+1) r} q^{(k+2)}\left(\begin{array}{l}r \\ 2\end{array}\right)\left[\begin{array}{l}n \\ r\end{array}\right] \frac{1-z^{2} q^{2 r-1}}{\left(z^{2} q^{r-1}\right)_{n+1}}$.

$$
\sum_{\ell(\lambda) \leq k} z^{|\lambda|} q^{2 n(\lambda)}\left[\begin{array}{l}
n \\
\lambda
\end{array}\right]=\sum_{r=0}^{n}(-1)^{r} z^{(k+1) r} q^{(2 k+3)}\left(\begin{array}{l}
r \\
2
\end{array}\right)\left[\begin{array}{l}
n \\
r
\end{array}\right] \frac{1-z q^{2 r-1}}{\left(z q^{r-1}\right)_{n+1}} .
$$

George Andrews [private communication] also obtained these identities as limiting cases of a generalization of Watson's Theorem [A4, Theorem 4].

Proof. If $w$ is any nontrivial permutation of $1, \ldots, n$, then the expression $\prod_{i<j}\left(x_{i}-q x_{j}\right)$ will vanish under the substitution $x_{i} \rightarrow z q^{w(i)-1}$. It follows that when $x_{i}=z q^{i-1}$, the only part of (1.3) that survives is the term indexed by the trivial permutation. After routine simplication of this single term (cf. Example III.2.1 of $[\mathrm{M}])$, one finds

$$
P_{\lambda^{\prime}}\left(z, z q, \ldots, z q^{n-1}\right)=z^{|\lambda|} q^{n(\lambda)}\left[\begin{array}{l}
n \\
\lambda
\end{array}\right] \text {. }
$$


Therefore, the coefficient of $u^{k}$ on the left in Theorem 1.2 becomes

$$
\sum_{\ell(\lambda) \leq k} z^{2|\lambda|} q^{2 n(\lambda)}\left[\begin{array}{l}
n \\
\lambda
\end{array}\right]
$$

Now consider the right side of Theorem 1.2. From the factorization in (1.10), it is clear that $\Psi(s x ; q)$ will vanish under the substitution $x_{i} \rightarrow z q^{i-1}$ if $x_{r} \in x_{-}$ and $x_{r-1} \in x_{+}$for some $r>0$. Hence, $\Psi(s x ; q)$ will be nonzero only if $x_{-}$ is of the form $\left(x_{1}, \ldots, x_{r}\right)$ for some $r$. In that case, it is easy to verify that $\Psi\left(x_{-}^{-1} ; q\right) \rightarrow(-1)^{r} z^{2 r} q^{3\left(\begin{array}{l}r \\ 2\end{array}\right)} /\left(z^{2} q^{r-1}\right)_{r}, \Psi\left(x_{+} ; q\right) \rightarrow 1 /\left(z^{2} q^{2 r}\right)_{n-r}$, and

$$
\prod_{x_{i} \in x_{-}, x_{j} \in x_{+}} \frac{1-q x_{j} x_{i}^{-1}}{1-x_{j} x_{i}^{-1}} \rightarrow\left[\begin{array}{l}
n \\
r
\end{array}\right]
$$

under the substitution $x_{i} \rightarrow z q^{i-1}$. We therefore have (cf. (1.10))

$$
\Psi(s x ; q) \rightarrow(-1)^{r} z^{2 r} q^{3}\left(\begin{array}{c}
r \\
2
\end{array}\right)\left[\begin{array}{l}
n \\
r
\end{array}\right] \frac{1-z^{2} q^{2 r-1}}{\left(z^{2} q^{r-1}\right)_{n+1}} .
$$

It follows that the coefficient of $u^{k}$ on the right in Theorem 1.2 becomes

$$
\sum_{r=0}^{n}(-1)^{r} z^{(2 k+2) r} q^{(2 k+3)\left(\begin{array}{l}
r \\
2
\end{array}\right)}\left[\begin{array}{l}
n \\
r
\end{array}\right] \frac{1-z^{2} q^{2 r-1}}{\left(z^{2} q^{r-1}\right)_{n+1}} .
$$

Identity (b) can now be obtained by substituting $z$ for $z^{2}$ throughout. Identity (a) follows similarly from Theorem 1.1 .

Taking the limit $n \rightarrow \infty$, we obtain

\section{Corollary 1.4 .}

$$
\sum_{\ell(\lambda) \leq k} z^{|\lambda|} \frac{q^{n(\lambda)}}{(q)_{\lambda}}=(-z)_{\infty} \sum_{r=0}^{\infty}(-1)^{r} z^{(k+1) r} q^{(k+2)}\left(\begin{array}{c}
r \\
2
\end{array}\right) \frac{1-z^{2} q^{2 r-1}}{(q)_{r}\left(z^{2} q^{r-1}\right)_{\infty}}
$$

$$
\sum_{\ell(\lambda) \leq k} z^{|\lambda|} \frac{q^{2 n(\lambda)}}{(q)_{\lambda}}=\sum_{r=0}^{\infty}(-1)^{r} z^{(k+1) r} q^{(2 k+3)\left(\begin{array}{l}
r \\
2
\end{array}\right)} \frac{1-z q^{2 r-1}}{(q)_{r}\left(z q^{r-1}\right)_{\infty}} .
$$

For both (a) and (b), there are two special values of $z$ for which the infinite series can be summed in closed form via Jacobi's Triple Product (0.1). For (b), the special values are $z=q$ and $z=q^{2}$. In the former case, the right side of (b) becomes

$$
\frac{1}{(q)_{\infty}}\left[1+\sum_{r=1}^{\infty}(-1)^{r} q^{(k+1) r+(2 k+3)\left(\begin{array}{l}
r \\
2
\end{array}\right)}\left(1+q^{r}\right)\right],
$$

and thus, aside from a multiplicative factor, it is of the form $(0.3)$ with the identifications $a \rightarrow q^{k+1}$ and $q \rightarrow q^{2 k+3}$. In the latter case, the right side of 
(b) becomes

$$
(q)_{\infty}^{-1} \sum_{r=0}^{\infty}(-1)^{r} q^{(2 k+2) r+(2 k+3)}\left(\begin{array}{l}
r \\
2
\end{array}\right)\left(1-q^{2 r+1}\right),
$$

and thus is of the form (0.4) with $a \rightarrow q^{2 k+2}$ and $q \rightarrow q^{2 k+3}$. Aside from multiplicative factors, one similarly finds that in case $z=q^{1 / 2}$, the right side of (a) assumes the form of (0.3) with $a \rightarrow q^{(k+1) / 2}, q \rightarrow q^{k+2}$, and in case $z=q$, it assumes the form of (0.4) with $a \rightarrow q^{k+1}, q \rightarrow q^{k+2}$.

The four $q$-series identities that these specializations produce are recorded below. The two that are consequences of Corollary 1.4(a) (and ultimately Theorem 1.1) are labeled (a) and (b); the two that are consequences of Corollary 1.4(b) and Theorem 1.2 are labeled (c) and (d). In one exceptional case (the second), we have substituted $q^{2}$ for $q$ to avoid fractional exponents. In each case, the infinite product is taken over all positive integers $n$ satisfying the indicated congruence.

\section{Corollary 1.5 .}

$$
\sum_{\ell(\lambda) \leq k} \frac{q^{n(\lambda)+|\lambda|}}{(q)_{\lambda}}=\frac{(-q)_{\infty}}{(q)_{\infty}} \prod\left(1-q^{n}\right), \quad n \equiv 0, \pm 1(\bmod k+2) ;
$$

(b) $\sum_{\ell(\lambda) \leq k} \frac{q^{2 n(\lambda)+|\lambda|}}{\left(q^{2} ; q^{2}\right)_{\lambda}}=\frac{\left(-q ; q^{2}\right)_{\infty}}{\left(q^{2} ; q^{2}\right)_{\infty}} \prod\left(1-q^{n}\right), \quad n \equiv 0, \pm(k+1)(\bmod 2 k+4)$;

$$
\sum_{\ell(\lambda) \leq k} \frac{q^{2 n(\lambda)+2|\lambda|}}{(q)_{\lambda}}=\frac{1}{(q)_{\infty}} \prod\left(1-q^{n}\right), \quad n \equiv 0, \pm 1(\bmod 2 k+3)
$$

$$
\sum_{\ell(\lambda) \leq k} \frac{q^{2 n(\lambda)+|\lambda|}}{(q)_{\lambda}}=\frac{1}{(q)_{\infty}} \prod\left(1-q^{n}\right), \quad n \equiv 0, \pm(k+1)(\bmod 2 k+3) .
$$

Note that the Rogers-Ramanujan identities are special cases of (c) and (d). (Take $k=1$.) Furthermore, (c) and (d) are themselves special cases of the following more general result due to Andrews [A2]:

$$
\sum_{\ell(\lambda) \leq k} \frac{q^{n_{2}(\lambda)+\lambda_{i}+\cdots+\lambda_{k}}}{(q)_{\lambda}}=\frac{1}{(q)_{\infty}} \prod_{n \equiv 0, \pm i(2 k+3)}\left(1-q^{n}\right),
$$

where $n_{2}(\lambda):=2 n(\lambda)+|\lambda|=\sum \lambda_{j}^{2}$, and $i$ and $k$ are any pair of integers satisfying $1 \leq i \leq k+1$. (Take $i=1$ and $i=k+1$ to obtain identities (c) and (d).)

In the special case $k=1$, identities (a) and (b) are equivalent to the special cases $z=q$ and $z=q^{1 / 2}$ of the following well-known result due to Euler [A5, 
(2.2.6)]:

$$
\sum_{n=0}^{\infty} \frac{z^{n} q^{\left(\begin{array}{l}
n \\
2
\end{array}\right)}}{(q)_{n}}=(-z)_{\infty}
$$

For general $k$, it seems that neither (a) nor (b) has previously appeared in the literature of $q$-series.

\section{ElEMENTARY PROOFS}

The $q$-series identities of the previous section were proved by starting with the Hall-Littlewood function identities (1.4) and (1.5), computing a partial fraction expansion à la Macdonald, and then specializing the variables. In this section, we will give a more elementary proof of the same identities by first specializing the variables, and then extracting partial fractions. We will also show that the specialized versions of (1.4) and (1.5) can be given elementary proofs, thus removing the need for the theory of Hall-Littlewood functions. In fact, it should be emphasized that the proof of Theorem 1.3 we give here, which includes Corollary 1.5 and the Rogers-Ramanujan identities among its consequences, is completely self-contained (aside from use of the easy-to-prove $q$-Binomial Identity $(0.2))$.

First, we record the identities obtained from (1.4) and (1.5) via the substitution $x_{i} \rightarrow z q^{i-1}$. With the aid of (1.11), it is easy to check that (1.4) becomes

$$
\sum_{\lambda} z^{|\lambda|} q^{n(\lambda)}\left[\begin{array}{l}
n \\
\lambda
\end{array}\right]=\frac{(-z)_{n}}{\left(z^{2}\right)_{n}}
$$

and similarly, after replacing $z^{2}$ with $z,(1.5)$ becomes

$$
\sum_{\lambda} z^{|\lambda|} q^{2 n(\lambda)}\left[\begin{array}{l}
n \\
\lambda
\end{array}\right]=\frac{1}{(z)_{n}}
$$

It is interesting to note that (2.2) is equivalent to an identity recently used by Stanton and Zeilberger to prove a conjecture of Odlyzko about the behavior of the formal power series $(1-q)^{k} /(q)_{k}[\mathrm{SZ},(\mathrm{JS})]$.

Elementary proof of (2.1). An application of the $q$-Binomial Identity (0.2) yields

$$
\frac{(-z)_{n}\left(z^{2} q^{n}\right)_{\infty}}{(-z)_{\infty}}=(-z)_{n} \sum_{r}(-z)^{r} \frac{\left(-z q^{n}\right)_{r}}{(q)_{r}}=\sum_{r}(-z)^{r} \frac{(-z)_{r}}{(q)_{r}}\left(-z q^{r}\right)_{n}
$$

Using $(0.5)$ to expand $\left(-z q^{r}\right)_{n}$, we obtain

$$
\begin{aligned}
& \frac{(-z)_{n}\left(z^{2} q^{n}\right)_{\infty}}{(-z)_{\infty}}=\sum_{r}(-z)^{r} \frac{(-z)_{r}}{(q)_{r}} \sum_{k}\left(z q^{r}\right)^{k} q^{\left(\begin{array}{c}
k \\
2
\end{array}\right)}\left[\begin{array}{l}
n \\
k
\end{array}\right] \\
& =\sum_{k} z^{k} q^{\left(\begin{array}{l}
k \\
2
\end{array}\right)}\left[\begin{array}{l}
n \\
k
\end{array}\right] \sum_{r}\left(-z q^{k}\right)^{r} \frac{(-z)_{r}}{(q)_{r}} \text {. }
\end{aligned}
$$


The inner sum on the right is of the form (0.2) with the identifications $a \rightarrow-z$ and $z \rightarrow-z q^{k}$, so it follows that

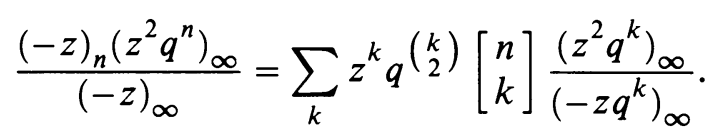

By cancelling the factor $\left(z^{2}\right)_{\infty} /(-z)_{\infty}$ from both sides we find

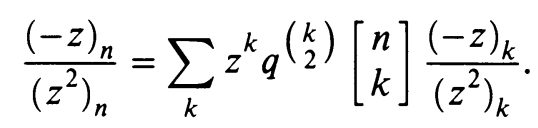

If we iterate this identity (and relabel the indices of summation), we obtain

$$
\frac{(-z)_{n}}{\left(z^{2}\right)_{n}}=\sum_{\lambda_{1}} z^{\lambda_{1}} q^{\left(\begin{array}{c}
\lambda_{1} \\
2
\end{array}\right)}\left[\begin{array}{c}
n \\
\lambda_{1}
\end{array}\right] \sum_{\lambda_{2}} z^{\lambda_{2}} q^{\left(\begin{array}{l}
\lambda_{2} \\
2
\end{array}\right)}\left[\begin{array}{l}
\lambda_{1} \\
\lambda_{2}
\end{array}\right] \frac{(-z)_{\lambda_{2}}}{\left(z^{2}\right)_{\lambda_{2}}},
$$

and if iterate the identity $l$ times, we obtain

$$
\frac{(-z)_{n}}{\left(z^{2}\right)_{n}}=\sum_{\ell(\lambda) \leq l} z^{|\lambda|} q^{n(\lambda)}\left[\begin{array}{l}
n \\
\lambda
\end{array}\right] \frac{(-z)_{\lambda_{l}}}{\left(z^{2}\right)_{\lambda_{l}}}
$$

The limit $l \rightarrow \infty$ yields $(2.1)$.

Elementary proof of (2.2). The following is a combinatorial argument; to find a similar proof of (2.1) appears to be a more difficult problem.

Recall that $1 /(q z)_{n}$ is the generating function for partitions of length at most $n$, with the partition $\mu$ being assigned the weight $z^{\mu_{1}} q^{|\mu|}$. By substituting $q z$ for $z$, it follows that $(2.2)$ is equivalent to the identity

$$
\sum_{\lambda} z^{|\lambda|} q^{n_{2}(\lambda)}\left[\begin{array}{l}
n \\
\lambda
\end{array}\right]=\sum_{\ell(\mu) \leq n} z^{\mu_{1}} q^{|\mu|},
$$

where $n_{2}(\lambda)=\lambda_{1}^{2}+\lambda_{2}^{2}+\cdots$, as in $\S 1$.

Let $\mu$ be a partition as above, identified with its Ferrers diagram; i.e., we identify $\mu$ with the set of lattice points $(i, j) \in \mathbf{Z}^{2}$ satisfying $1 \leq i \leq \ell(\mu)$, $1 \leq j \leq \mu_{i}$. Let $\lambda_{1}$ denote the length of the main diagonal of $\mu$. If we delete the $\lambda_{1} \times \lambda_{1}$ square subdiagram of $\mu$, the remainder will consist (modulo translation) of a partition $\mu_{L}$ ( $L$ for "leg") contained in an $\left(n-\lambda_{1}\right) \times \lambda_{1}$ rectangle, and a partition $\mu_{A}$ ( $A$ for "arm") of length at most $\lambda_{1}$. Continuing this process, let $\lambda_{2}$ denote the length of the main diagonal of $\mu_{A}$, with $\mu_{A L}$ and $\mu_{A A}$ denoting the partitions that remain after deletion of the $\lambda_{2} \times \lambda_{2}$ square subdiagram from $\mu_{A}$. Note that $\mu_{A L}$ is contained in a $\left(\lambda_{1}-\lambda_{2}\right) \times \lambda_{2}$ rectangle, and $\mu_{A A}$ has length at most $\lambda_{2}$.

If we repeat this process indefinitely, we obtain a partition $\lambda=\left(\lambda_{1} \geq \lambda_{2} \geq \cdots\right)$ and a sequence of partitions $\mu_{L}, \mu_{A L}, \mu_{A A L}, \ldots$ contained in rectangles of dimension $\left(\lambda_{i}-\lambda_{i+1}\right) \times \lambda_{i+1}\left(i=0,1,2, \ldots ; \lambda_{0}=n\right)$, respectively. The process is clearly invertible. An example appears in Figure 1. 


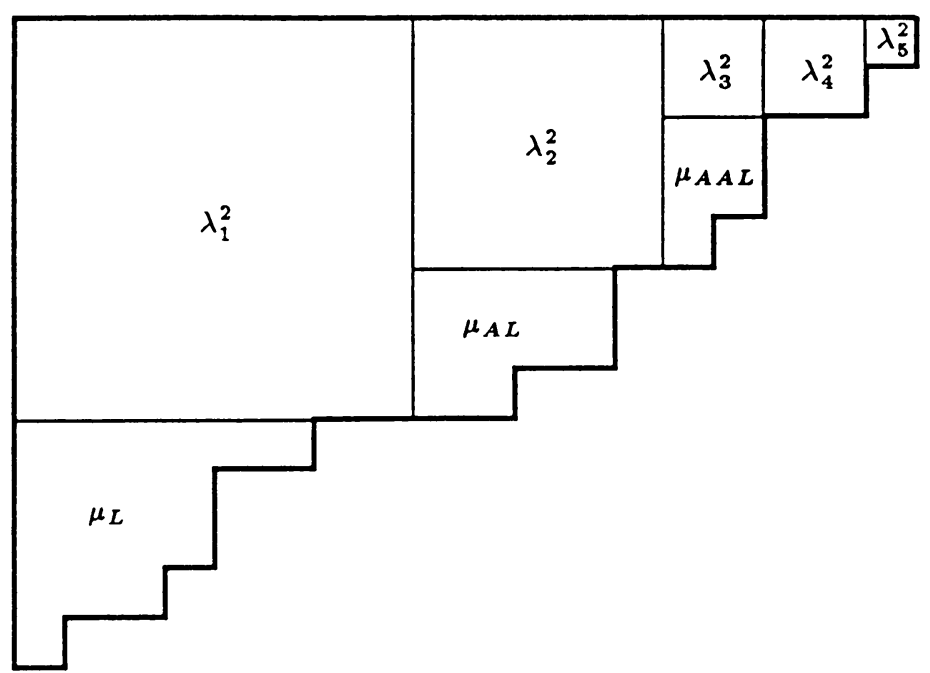

FIGURE 1

Observe that $\mu_{1}=|\lambda|$ and $|\mu|=n_{2}(\lambda)+\left|\mu_{L}\right|+\left|\mu_{A L}\right|+\cdots$. Since the generating function for partitions contained in a $k \times l$ rectangle is $\left[\begin{array}{c}k+l \\ k\end{array}\right]$ [A5, 3.2], it follows that the generating function for the partitions $\mu$ corresponding to a fixed $\lambda$ is of the form

$$
z^{|\lambda|} q^{n_{2}(\lambda)}\left[\begin{array}{l}
n \\
\lambda_{1}
\end{array}\right]\left[\begin{array}{l}
\lambda_{1} \\
\lambda_{2}
\end{array}\right]\left[\begin{array}{l}
\lambda_{2} \\
\lambda_{3}
\end{array}\right] \cdots=z^{|\lambda|} q^{n_{2}(\lambda)}\left[\begin{array}{l}
n \\
\lambda
\end{array}\right] .
$$

Identity (2.2) follows upon summation over the choices for $\lambda$. We remark that a similar proof technique appeared in [A6].

Elementary proof of Theorem 1.3. By analogy with the proof of Theorem 1.2, we define

$$
\psi(u)=\sum_{k>0} u^{k} \sum_{\ell(\lambda) \leq k} z^{|\lambda|} q^{2 n(\lambda)}\left[\begin{array}{l}
n \\
\lambda
\end{array}\right]=\frac{1}{1-u} \sum_{\lambda} z^{|\lambda|} q^{2 n(\lambda)} u^{\ell(\lambda)}\left[\begin{array}{l}
n \\
\lambda
\end{array}\right]
$$

For any partition $\lambda$ with terms $\leq n$, let $\nu_{1}, \ldots, \nu_{l}$ denote the set of distinct parts other than $n$ occurring in $\lambda$, ordered so that $n>\nu_{1}>\cdots>\nu_{l}>0$. Whether or not $n$ actually occurs as a part of $\lambda$, it is convenient to define $\nu_{0}=n$. Let $m_{i}$ denote the multiplicity of $\nu_{i}$ in $\lambda$, with the convention that $m_{0}=0$ if $n$ does not occur in $\lambda$. In terms of these parameters, we have $\left[\begin{array}{l}n \\ \lambda\end{array}\right]=\left[\begin{array}{l}n \\ \nu\end{array}\right]$ and

$$
\begin{gathered}
\ell(\lambda)=m_{0}+\cdots+m_{l}, \quad|\lambda|=\nu_{0} m_{0}+\cdots \nu_{l} m_{l}, \\
n(\lambda)=\left(\begin{array}{c}
\nu_{0} \\
2
\end{array}\right) m_{0}+\cdots+\left(\begin{array}{c}
\nu_{l} \\
2
\end{array}\right) m_{l} .
\end{gathered}
$$


Therefore, (2.3) can be rewritten in the form (cf. (1.7))

$$
\begin{aligned}
\psi(u) & =\frac{1}{1-u} \sum_{(m), \nu}\left[\begin{array}{l}
n \\
\nu
\end{array}\right]\left(z^{\nu_{0}} q^{\nu_{0}\left(\nu_{0}-1\right)} u\right)^{m_{0}} \cdots\left(z^{\nu_{l}} q^{\nu_{l}\left(\nu_{l}-1\right)} u\right)^{m_{l}} \\
& =\frac{1}{1-u} \sum_{\nu}\left[\begin{array}{l}
n \\
\nu
\end{array}\right]\left\langle z^{\nu_{1}} q^{\nu_{1}\left(\nu_{1}-1\right)} u\right\rangle \cdots\left\langle z^{\nu_{l}} q^{\nu_{l}\left(\nu_{l}-1\right)} u\right\rangle \frac{1}{1-u z^{n} q^{n(n-1)}} .
\end{aligned}
$$

This expansion shows that $\psi$ is a rational function of $u$ with simple poles occurring at $u=z^{-r} q^{-r(r-1)}(0 \leq r \leq n)$. Since $\psi(u)$ vanishes at infinity, there must be a partial fraction decomposition of the form

$$
\psi(u)=\sum_{r=0}^{n} \frac{c_{r}}{1-u z^{r} q^{r(r-1)}},
$$

where $c_{r}=c_{r}^{(n)}(z, q)$ denotes the limit of

$$
\psi(u)\left(1-u z^{r} q^{r(r-1)}\right)
$$

as $u \rightarrow z^{-r} q^{-r(r-1)}$.

From the definition of $\psi(u)$ and (2.2), we have $c_{0}=1 /(z)_{n}$, so in view of (2.4), one may deduce

$$
\frac{1}{(z)_{n}}=\frac{1}{1-z^{n} q^{n(n-1)}} \sum_{\nu}\left[\begin{array}{l}
n \\
\nu
\end{array}\right]\left\langle z^{\nu_{1}} q^{\nu_{1}\left(\nu_{1}-1\right)}\right\rangle \cdots\left\langle z^{\nu_{l}} q^{\nu_{l}\left(\nu_{l}-1\right)}\right\rangle
$$

Substituting $z^{-1} q^{-2(n-1)}$ for $z$, it follows that

$$
\begin{aligned}
\frac{1}{\left(z^{-1} q^{-2(n-1)}\right)_{n}} & =\frac{1}{1-z^{-n} q^{-n(n-1)}} \sum_{\nu}\left[\begin{array}{l}
n \\
\nu
\end{array}\right] \prod_{i}\left\langle z^{-\nu_{i}} q^{-2 \nu_{i}(n-1)+\nu_{i}\left(\nu_{i}-1\right)}\right\rangle \\
& =\frac{1}{1-z^{-n} q^{-n(n-1)}} \sum_{\mu}\left[\begin{array}{l}
n \\
\mu
\end{array}\right] \prod_{i}\left\langle z^{\mu_{i}-n} q^{\mu_{i}\left(\mu_{i}-1\right)-n(n-1)}\right\rangle,
\end{aligned}
$$

where $\mu_{i}=n-\nu_{l+1-i}(1 \leq i \leq l)$. This latter calculation, together with (2.4), shows that $c_{n}=1 /\left(z^{-1} q^{-2(n-1)}\right)_{n}$.

Finally, to compute $c_{r}(0<r<n)$, note that a generic term of (2.4) will contribute to $c_{r}$ only if $r$ is one of the terms of $\nu$; say $\nu_{j}=r$. For each such sequence $\nu$, define $\rho_{i}=\nu_{i}-r(0 \leq i<j)$ and $\sigma_{i}=\nu_{i+j}(0 \leq i \leq l-j)$, and observe that the terms of the sequences $\rho$ and $\sigma$ are bounded by $n-r$ and $r$, respectively. Rewriting the contributions in terms of these new parameters, we see that $(2.4)$ implies

$$
\begin{aligned}
c_{r}\left[\begin{array}{l}
n \\
r
\end{array}\right]^{-1}= & \frac{1}{1-z^{n-r} q^{n(n-1)-r(r-1)}} \sum_{\rho}\left[\begin{array}{c}
n-r \\
\rho
\end{array}\right] \prod_{i}\left\langle z^{\rho_{i}} q^{\left(\rho_{i}+r\right)\left(\rho_{i}+r-1\right)-(r-1)}\right\rangle \\
& \cdot \frac{1}{1-z^{-r} q^{-r(r-1)}} \sum_{\sigma}\left[\begin{array}{c}
r \\
\sigma
\end{array}\right] \prod_{i}\left\langle z^{\sigma_{i}-r} q^{\sigma_{i}\left(\sigma_{i}-1\right)-r(r-1)}\right\rangle,
\end{aligned}
$$


and therefore, by (2.5) and (2.6),

$$
c_{r}=\left[\begin{array}{l}
n \\
r
\end{array}\right] \cdot \frac{1}{\left(z q^{2 r}\right)_{n-r}\left(z^{-1} q^{-2(r-1)}\right)_{r}}=(-1)^{r} z^{r} q^{3\left(\begin{array}{l}
r \\
2
\end{array}\right)}\left[\begin{array}{l}
n \\
r
\end{array}\right] \frac{1-z q^{2 r-1}}{\left(z q^{r-1}\right)_{n+1}} .
$$

This completes the calculation of the partial fractions, so we now have

$$
\psi(u)=\sum_{r=0}^{n}(-1)^{r} z^{r} q^{3\left(\begin{array}{l}
r \\
2
\end{array}\right)}\left[\begin{array}{l}
n \\
r
\end{array}\right] \frac{1-z q^{2 r-1}}{\left(z q^{r-1}\right)_{n+1}} \frac{1}{1-u z^{r} q^{r(r-1)}} .
$$

Theorem 1.3(b) now follows upon comparison with (2.3).

A proof of Theorem 1.3(a) can be obtained by a similar argument. We define

$$
\varphi(u)=\sum_{k \geq 0} u^{k} \sum_{\ell(\lambda) \leq k} z^{|\lambda|} q^{n(\lambda)}\left[\begin{array}{l}
n \\
\lambda
\end{array}\right]=\frac{1}{1-u} \sum_{\lambda} z^{|\lambda|} q^{n(\lambda)} u^{\ell(\lambda)}\left[\begin{array}{l}
n \\
\lambda
\end{array}\right],
$$

and note that in terms of the parameter $\nu$, we have

$$
\varphi(u)=\frac{1}{1-u} \sum_{\nu}\left[\begin{array}{l}
n \\
\nu
\end{array}\right] \prod_{i}\left\langle z^{\nu_{i}} q^{\left(\begin{array}{c}
\nu_{i} \\
2
\end{array}\right)} u\right\rangle \frac{1}{1-u z^{n} q^{\left(\begin{array}{c}
n \\
2
\end{array}\right)}} .
$$

This expansion shows that $\varphi$ is a rational function of $u$ with a partial fraction decomposition of the form

$$
\varphi(u)=\sum_{r=0}^{n} \frac{b_{r}}{1-u z^{r} q^{\left(\begin{array}{r}
r \\
2
\end{array}\right)}},
$$

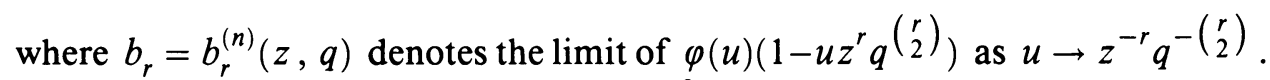

Note that $(2.1)$ implies $b_{0}=(-z)_{n} /\left(z^{2}\right)_{n}$. If we use (2.8) to calculate the

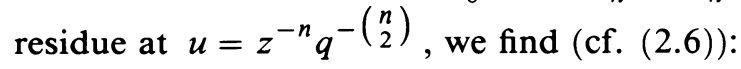

$$
b_{n}^{(n)}(z, q)=b_{0}^{(n)}\left(z^{-1} q^{-(n-1)}, q\right)=(-1)^{n} z^{n} q^{2\left(\begin{array}{c}
n \\
2
\end{array}\right)} \frac{(-z)_{n}}{\left(z^{2} q^{(n-1)}\right)_{n}},
$$

and more generally (cf. (2.7)),

$$
b_{r}^{(n)}(z, q)=\left[\begin{array}{l}
n \\
r
\end{array}\right] b_{r}^{(r)}(z, q) b_{0}^{(n-r)}\left(z q^{r}, q\right)=(-1)^{r} z^{r} q^{2\left(\begin{array}{c}
r \\
2
\end{array}\right)} \frac{(-z)_{n}\left(1-z^{2} q^{2 r-1}\right)}{\left(z^{2} q^{r-1}\right)_{n+1}} .
$$

We therefore have

$$
\varphi(u)=\sum_{r=0}^{n}(-1)^{r} z^{r} q^{2\left(\begin{array}{c}
r \\
2
\end{array}\right)}\left[\begin{array}{l}
n \\
r
\end{array}\right] \frac{(-z)_{n}}{\left(z^{2} q^{r-1}\right)_{n+1}} \cdot \frac{1-z^{2} q^{2 r-1}}{1-u z^{r} q^{\left(\begin{array}{c}
r \\
2
\end{array}\right)}},
$$

and hence we obtain Theorem 1.3(a) by examining the coefficient of $u^{k}$.

\section{Generalizations}

In the previous section we reproved the identities of Theorem 1.3 by applying Macdonald's partial fraction method directly to the $q$-series (2.1) and (2.2), 
rather than the Hall-Littlewood function identities they arose from. In this section, we will prove some more general Hall-Littlewood identities, specialize the variables to obtain $q$-series, and then follow the partial fraction method as before. In this more general context, the partial fraction expansions analogous to those in Theorems 1.1 and 1.2 do not have a simple form; it is only after specialization that explicit results become feasible.

Let $x=\left(x_{1}, \ldots, x_{n}\right)$ and $y=\left(y_{1}, \ldots, y_{m}\right)$ be two (distinct) collections of independent indeterminates. If $f$ is a symmetric function of $n+m$ variables, we use the notation $f(x, y)$ as an abbreviation for $f\left(x_{1}, \ldots, x_{n}, y_{1}, \ldots, y_{m}\right)$. Since $P_{\lambda}(x, y ; q)$ can be viewed as a function independently symmetric with respect to $x$ and $y$, it follows that the coefficient of $P_{\mu}(x ; q)$ in $P_{\lambda}(x, y ; q)$ is a symmetric function of $y$. This coefficient is the skew Hall-Littlewood function $P_{\lambda / \mu}(y ; q)[\mathrm{M}, \mathrm{III} .5]$, and is thus characterized by the expansion

$$
P_{\lambda}(x, y ; q)=\sum_{\mu} P_{\mu}(x ; q) P_{\lambda / \mu}(y ; q) .
$$

We remark that it can be shown that $P_{\lambda / \mu}=0$ unless $\mu \subseteq \lambda$; i.e., the diagram of $\lambda$ must contain the diagram of $\mu$ [M, III.(5.3)].

Let $Q_{\lambda}$ and $Q_{\lambda / \mu}$ denote the following scalar multiples of $P_{\lambda}$ and $P_{\lambda / \mu}$ :

$$
\begin{aligned}
Q_{\lambda}(x ; q) & =(q)_{\lambda^{\prime}} P_{\lambda}(x ; q), \\
Q_{\lambda / \mu}(x ; q) & =\frac{(q)_{\lambda^{\prime}}}{(q)_{\mu^{\prime}}} P_{\lambda / \mu}(x ; q),
\end{aligned}
$$

and note that (3.1) also holds with $Q$ in place of $P$. These " $Q$-functions" appear conveniently in the following generating function due to Littlewood [M, III.4]:

$$
\sum_{\lambda} P_{\lambda}(x ; q) Q_{\lambda}(y ; q)=\prod_{i, j} \frac{1-q x_{i} y_{j}}{1-x_{i} y_{j}} .
$$

In case $q=0$, this specializes to the Cauchy identity for Schur functions [M, I.4].

The following pair of Hall-Littlewood function identities will be used to derive (via the usual specialization) $q$-series identities that generalize $(2.1)$ and (2.2).

Lemma 3.1. Let $\alpha$ and $\beta$ denote fixed partitions.

$$
\begin{gathered}
\text { (a) } \sum_{\lambda} P_{\lambda / \alpha}(x ; q)=\sum_{\mu} Q_{\alpha / \mu}(x ; q) \prod_{i} \frac{1}{1-x_{i}} \prod_{i<j} \frac{1-q x_{i} x_{j}}{1-x_{i} x_{j}} . \\
\text { (b) } \sum_{\lambda} P_{\lambda / \alpha}(x ; q) Q_{\lambda / \beta}(y ; q)=\sum_{\mu} P_{\beta / \mu}(x ; q) Q_{\alpha / \mu}(y ; q) \prod_{i, j} \frac{1-q x_{i} y_{j}}{1-x_{i} y_{j}} .
\end{gathered}
$$

We remark that these identities (and their proofs) are well known, but except for special cases, they do not seem to have appeared in the literature of symmetric functions. A proof of the special case $q=0$ (i.e., the corresponding Schur 
function identities) can be found in A. V. Zelevinsky's Russian translation of [M], and combinatorial proofs of the special cases $q=0$ and $q=-1$ can be found in [SS]. Note that identities (1.4) and (3.2) are also special cases of this result.

Proof. For each partition $\beta$, define

$$
F_{\beta}(x, y)=\sum_{\lambda} P_{\lambda}(x ; q) Q_{\lambda / \beta}(y ; q) .
$$

By introducing a third indeterminate set $z=\left(z_{1}, \ldots, z_{m}\right)$, an application of (3.2) and the $Q$ version of (3.1) will yield

$$
\begin{aligned}
& \sum_{\beta} F_{\beta}(x, y) Q_{\beta}(z ; q)=\sum_{\lambda} P_{\lambda}(x ; q) Q_{\lambda}(z, y ; q) \\
& =\prod_{i, j} \frac{1-q x_{i} y_{j}}{1-x_{i} y_{j}} \frac{1-q x_{i} z_{j}}{1-x_{i} z_{j}} .
\end{aligned}
$$

Therefore, a second application of (3.2) in the variables $x$ and $z$ implies

$$
\sum_{\beta} F_{\beta}(x, y) Q_{\beta}(z ; q)=\sum_{\gamma} P_{\gamma}(x ; q) Q_{\gamma}(z ; q) \prod_{i, j} \frac{1-q x_{i} y_{j}}{1-x_{i} y_{j}} .
$$

After comparing coefficients of $Q_{\beta}(z ; q)$, we conclude that

$$
\sum_{\lambda} P_{\lambda}(x ; q) Q_{\lambda / \beta}(y ; q)=P_{\beta}(x ; q) \prod_{i, j} \frac{1-q x_{i} y_{j}}{1-x_{i} y_{j}} .
$$

This may be recognized as the special case $\alpha=\varnothing$ of (b).

Now consider the more general series

$$
F_{\alpha \beta}(x, y)=\sum_{\lambda} P_{\lambda / \alpha}(x ; q) Q_{\lambda / \beta}(y ; q) .
$$

An application of (3.1) and (3.3) yields

$$
\begin{gathered}
\sum_{\alpha} P_{\alpha}(z) F_{\alpha \beta}(x, y)=\sum_{\lambda} P_{\lambda}(z, x) Q_{\lambda / \beta}(y) \\
=P_{\beta}(z, x) \prod_{i, j} \frac{1-q z_{i} y_{j}}{1-z_{i} y_{j}} \frac{1-q x_{i} y_{j}}{1-x_{i} y_{j}} .
\end{gathered}
$$

A further application of $(3.1)$ to $P_{\beta}(z, x ; q)$ therefore shows that $F_{\alpha \beta}(x, y)$ is the coefficient of $P_{\alpha}(z)$ in

$$
\sum_{\mu} P_{\mu}(z) P_{\beta / \mu}(x) \prod_{i, j} \frac{1-q z_{i} y_{j}}{1-z_{i} y_{j}} \frac{1-q x_{i} y_{j}}{1-x_{i} y_{j}} .
$$

However, (3.3) shows that the coefficient of $P_{\alpha}(z)$ in

$$
P_{\mu}(z) \prod\left(1-q z_{i} y_{j}\right) /\left(1-z_{i} y_{j}\right)
$$


is $Q_{\alpha / \mu}(y)$, and so we have

$$
F_{\alpha \beta}(x, y)=\sum_{\mu} P_{\beta / \mu}(x ; q) Q_{\alpha / \mu}(y ; q) \prod_{i, j} \frac{1-q x_{i} y_{j}}{1-x_{i} y_{j}},
$$

thus completing the proof of $(b)$.

To prove (a), define $G_{\alpha}(x)=\sum_{\lambda} P_{\lambda / \alpha}(x ; q)$, and observe that (1.4) and (3.1) imply

$$
\begin{aligned}
& \sum_{\alpha} G_{\alpha}(x) P_{\alpha}(y)=\sum_{\lambda} P_{\lambda}(y, x) \\
& \quad=\prod_{i} \frac{1}{1-x_{i}} \frac{1}{1-y_{i}} \prod_{i<j} \frac{1-q x_{i} x_{j}}{1-x_{i} x_{j}} \frac{1-q y_{i} y_{j}}{1-y_{i} y_{j}} \prod_{i, j} \frac{1-q x_{i} y_{j}}{1-x_{i} y_{j}} .
\end{aligned}
$$

Therefore, $G_{\alpha}(x)$ is the coefficient of $P_{\alpha}(y)$ in

$$
\sum_{\mu} P_{\mu}(y) \prod_{i, j} \frac{1-q x_{i} y_{j}}{1-x_{i} y_{j}} \prod_{i} \frac{1}{1-x_{i}} \prod_{i<j} \frac{1-q x_{i} x_{j}}{1-x_{i} x_{j}} .
$$

However, (3.3) shows that the coefficient of $P_{\alpha}(y)$ in

$$
P_{\mu}(y) \prod\left(1-q x_{i} y_{j}\right) /\left(1-x_{i} y_{j}\right)
$$

is $Q_{\alpha / \mu}(x)$, and so we have

$$
G_{\alpha}(x)=\sum_{\mu} Q_{\alpha / \mu}(x ; q) \prod_{i} \frac{1}{1-x_{i}} \prod_{i<j} \frac{1-q x_{i} x_{j}}{1-x_{i} x_{j}},
$$

which completes the proof of (a).

In order to use Lemma 3.1 to prove $q$-series identities, we need information about the specializations $x_{i} \rightarrow q^{i-1}$ of $P_{\lambda / \alpha}(x ; q)$. In general, these specializations do not factor into a simple closed form. However, the following result shows that in case $\alpha=1^{r}$ (the partition consisting of $r 1$ 's), there is a simple factorization in the limit $n \rightarrow \infty$.

Lemma 3.2.

$$
\begin{aligned}
& Q_{\lambda / 1^{r}}\left(1, q, q^{2}, \ldots ; q\right)=q^{n\left(\lambda^{\prime}\right)-\left(\begin{array}{c}
r \\
2
\end{array}\right)}\left[\begin{array}{c}
\ell(\lambda) \\
r
\end{array}\right]\left(q^{-1}\right) \\
& \sum_{r} z^{r} Q_{\lambda / 1^{r}}\left(1, q, q^{2}, \ldots ; q\right)=q^{n\left(\lambda^{\prime}\right)}\left(-z ; q^{-1}\right)_{\ell(\lambda)} .
\end{aligned}
$$

We remark that this result can be obtained as a corollary of Example III.3.1 of $[\mathrm{M}]$, although the proof given there relies on the deep connection between Hall-Littlewood functions and the structure of modules over discrete valuation rings with finite residue field. The following proof does not require this extra apparatus. 
Proof. Note that (0.5) shows that (a) and (b) are equivalent, so it suffices to establish (b). We next observe that in the limit $n \rightarrow \infty,(1.11)$ implies $P_{\mu}\left(1, q, q^{2}, \ldots ; q\right)=q^{n\left(\mu^{\prime}\right)} /(q)_{\mu^{\prime}}$, and therefore $Q_{\mu}\left(1, q, q^{2}, \ldots ; q\right)=q^{n\left(\mu^{\prime}\right)}$. It follows that

$$
\sum_{r} z^{r} Q_{\lambda / 1^{r}}\left(1, q, q^{2}, \ldots ; q\right)=q^{n\left(\lambda^{\prime}\right)} \sum_{r, \mu} z^{r} q^{n\left(\mu^{\prime}\right)-n\left(\lambda^{\prime}\right)} f_{\mu, r^{r}}^{\lambda}(q),
$$

where $f_{\mu, 1^{r}}^{\lambda}(q)$ denotes the coefficient of $Q_{\mu}$ in $Q_{\lambda / 1^{r}}$. According to [M, III.(3.2)], we have $f_{\mu, 1^{r}}^{\lambda}(q)=0$ unless $\mu$ can be obtained by reducing $r$ of the parts of $\lambda$ by 1 . In that case, assuming that (1) the distinct terms of $\lambda$ are $\nu_{1}>\cdots>\nu_{k}>0$, (2) the multiplicity of $\nu_{i}$ in $\lambda$ is $m_{i}$, and (3) $r_{i}$ of the terms $\nu_{i}$ in $\lambda$ need to be reduced by 1 to obtain $\mu$, then we have (loc. cit.):

$$
f_{\mu, 1^{r}}^{\lambda}(q)=\prod_{i}\left[\begin{array}{c}
m_{i} \\
r_{i}
\end{array}\right]=\prod_{i} q^{r_{i}\left(m_{i}-r_{i}\right)}\left[\begin{array}{c}
m_{i} \\
r_{i}
\end{array}\right]\left(q^{-1}\right) .
$$

If we define $s_{i}=m_{1}+\cdots+m_{i-1}$, then $n\left(\mu^{\prime}\right)-n\left(\lambda^{\prime}\right)=\sum_{i}\left(\begin{array}{c}s_{i}+m_{i}-r_{i} \\ 2\end{array}\right)-\left(\begin{array}{c}s_{i}+m_{i} \\ 2\end{array}\right)=-\sum_{i} r_{i} s_{i}+r_{i}\left(m_{i}-r_{i}\right)+\left(\begin{array}{c}r_{i} \\ 2\end{array}\right)$, and so we can rewrite the form of (3.4) and apply (0.5) to deduce

$$
\begin{aligned}
\sum_{r} z^{r} Q_{\lambda / 1^{r}}\left(1, q, q^{2}, \ldots ; q\right) & =q^{n\left(\lambda^{\prime}\right)} \prod_{i=1}^{k} \sum_{r_{i}} z^{r_{i}} q^{-r_{i} s_{i}-\left(\begin{array}{c}
r_{i} \\
2
\end{array}\right)}\left[\begin{array}{c}
m_{i} \\
r_{i}
\end{array}\right]\left(q^{-1}\right) \\
& =q^{n\left(\lambda^{\prime}\right)} \prod_{i=1}^{k}\left(-z q^{-s_{i}} ; q^{-1}\right)_{m_{i}}=\left(-z ; q^{-1}\right)_{\ell(\lambda)} .
\end{aligned}
$$

We now specialize the identities in Lemma 3.1 to obtain generalizations of the limiting case $n \rightarrow \infty$ of (2.1) and (2.2).

\section{Lemma 3.3.}

$$
\begin{gathered}
\sum_{\lambda} z^{|\lambda|} q^{n(\lambda)} \frac{\left(a ; q^{-1}\right)_{\lambda_{1}}}{(q)_{\lambda}}=\frac{(-z)_{\infty}\left(a z^{2}\right)_{\infty}}{\left(z^{2}\right)_{\infty}(-a z)_{\infty}} \\
\sum_{\lambda} z^{|\lambda|} q^{2 n(\lambda)} \frac{\left(a ; q^{-1}\right)_{\lambda_{1}}\left(b ; q^{-1}\right)_{\lambda_{1}}}{(q)_{\lambda}}=\frac{(a z)_{\infty}(b z)_{\infty}}{(z)_{\infty}(a b z)_{\infty}} .
\end{gathered}
$$

Proof. In the special case $\alpha=1^{r}, \beta=1^{s}$, Lemma 3.1(b) implies

$$
\sum_{\lambda} P_{\lambda / 1^{r}}(x ; q) Q_{\lambda / 1^{s}}(y ; q)=\prod_{i, j} \frac{1-q x_{i} y_{j}}{1-x_{i} y_{j}} \sum_{\mu} P_{1^{s} / \mu}(x ; q) Q_{1^{r} / \mu}(y ; q),
$$

and therefore, since $P_{\lambda / \mu}=0$ unless $\mu \subseteq \lambda$,

$$
\sum_{\lambda} \frac{(q)_{r}}{(q)_{\lambda}} Q_{\lambda^{\prime} / 1^{r}}(x ; q) Q_{\lambda^{\prime} / 1^{s}}(y ; q)=\prod_{i, j} \frac{1-q x_{i} y_{j}}{1-x_{i} y_{j}} \sum_{t} \frac{(q)_{t}}{(q)_{s}} Q_{1^{s} / 1^{\prime}}(x ; q) Q_{1^{r} / 1^{t}}(y ; q) \text {. }
$$


If we set $x_{i}=z q^{i-1}$ and $y_{i}=q^{i-1}$, then we may apply Lemma 3.2 to each of the four $Q$-functions appearing above, and thereby deduce

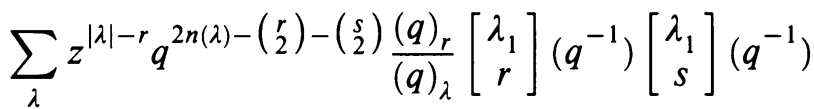

$$
\begin{aligned}
& =\frac{1}{(z)_{\infty}} \sum_{t} z^{s-t} \frac{(q)_{r}}{(q)_{t}} \frac{\left.\left.q^{(s-t}\right)^{(}\right)}{(q)_{s-t}} \frac{\left.q^{(r-t} 2\right)}{(q)_{r-t}},
\end{aligned}
$$

in the limit $n \rightarrow \infty$. Finally, rescale this identity by $(-a z)^{r}(-b)^{s} /(q)_{r}$, sum over $r, s \geq 0$, and apply the $q$-Binomial Identities $(0.2)$ and $(0.5)$ to obtain

$$
\begin{aligned}
& \sum_{\lambda} z^{|\lambda|} q^{2 n(\lambda)} \frac{\left(a ; q^{-1}\right)_{\lambda_{1}}\left(b ; q^{-1}\right)_{\lambda_{1}}}{(q)_{\lambda}} \\
& =\frac{1}{(z)_{\infty}} \sum_{r, s \geq t} \frac{(a b z)^{t}}{(q)_{t}} \frac{\left.(-b z)^{s-t} q^{(s-t}{ }^{2}\right)}{(q)_{s-t}} \frac{(-a z)^{r-t} q^{\left(\frac{r-t}{2}\right)}}{(q)_{r-t}} \\
& =\frac{(a z)_{\infty}(b z)_{\infty}}{(z)_{\infty}} \sum_{t} \frac{(a b z)^{t}}{(q)_{t}}=\frac{(a z)_{\infty}(b z)_{\infty}}{(z)_{\infty}(a b z)_{\infty}}
\end{aligned}
$$

as desired. (To obtain the second equality, apply the limiting case $n \rightarrow \infty$ of (0.5).)

To prove (a), start with the special case $\alpha=1^{r}$ of Lemma 3.1(a), specialize the variables, and proceed as above.

We remark that it is possible to prove Lemma 3.3 via elementary methods analogous to those of $\S 2$. For example, to prove (a), let $k=\lambda_{1}, \mu=$ $\left(\lambda_{2}, \lambda_{3}, \ldots\right)$, so that $\mu$ is a partition with $\mu_{1} \leq k$. We then have

$$
\begin{aligned}
\sum_{\lambda} z^{|\lambda|} q^{n(\lambda)} \frac{\left(a ; q^{-1}\right) \lambda_{1}}{(q)_{\lambda}} & =\sum_{k} z^{k} q^{\left(\frac{k}{2}\right)} \frac{\left(a ; q^{-1}\right)_{k}}{(q)_{k}} \sum_{\mu} z^{|\mu|} q^{n(\mu)}\left[\begin{array}{l}
k \\
\mu
\end{array}\right] \\
& =\sum_{k}(-a z)^{k} \frac{\left(a^{-1}\right)_{k}(-z)_{k}}{(q)_{k}\left(z^{2}\right)_{k}}
\end{aligned}
$$

after an application of (2.1). This latter expression is in the standard form of a ${ }_{2} \varphi_{1}$ basic hypergeometric series; by applying Heine's transformation [A9, (2.11)], one easily obtains the desired result.

We are now ready to prove a generalization of Theorem 1.3 in the limit $n \rightarrow \infty$ (cf. Corollary 1.4). George Andrews [private communication] also obtained these two results as limiting cases of a generalization of Watson's Theorem [A4, Theorem 4]. 


\section{Theorem 3.4.}

(a) $\sum_{\ell(\lambda) \leq k} z^{|\lambda|} q^{n(\lambda)} \frac{\left(a ; q^{-1}\right)_{\lambda_{1}}}{(q)_{\lambda}}$

$$
=\frac{(-z)_{\infty}}{(-a z)_{\infty}} \sum_{r=0}^{\infty}(-1)^{r} z^{(k+1) r} q^{(k+2)\left(\begin{array}{c}
r \\
2
\end{array}\right)} \frac{\left(a ; q^{-1}\right)_{r}\left(a z^{2} q^{r}\right)_{\infty}\left(1-z^{2} q^{2 r-1}\right)}{(q)_{r}\left(z^{2} q^{r-1}\right)_{\infty}} .
$$

(b)

$$
\begin{aligned}
\sum_{\ell(\lambda) \leq k} z^{|\lambda|} q^{2 n(\lambda)} \frac{\left(a ; q^{-1}\right)_{\lambda_{1}}\left(b ; q^{-1}\right)_{\lambda_{1}}}{(q)_{\lambda}} & \\
=\sum_{r=0}^{\infty}(-1)^{r} z^{(k+1) r} q^{(2 k+3)\left(\begin{array}{l}
r \\
2
\end{array}\right)} & \\
& \cdot \frac{\left(a ; q^{-1}\right)_{r}\left(b ; q^{-1}\right)_{r}\left(a z q^{r}\right)_{\infty}\left(b z q^{r}\right)_{\infty}\left(1-z q^{2 r-1}\right)}{(q)_{r}\left(z q^{r-1}\right)_{\infty}(a b z)_{\infty}}
\end{aligned}
$$

Proof. Following the methods of $\S 2$, we define

$$
\begin{aligned}
\psi_{a b}(u) & =\sum_{k \geq 0} u^{k} \sum_{\ell(\lambda) \leq k} z^{|\lambda|} q^{2 n(\lambda)} \frac{\left(a ; q^{-1}\right)_{\lambda_{1}}\left(b ; q^{-1}\right)_{\lambda_{1}}}{(q)_{\lambda}} \\
& =\frac{1}{1-u} \sum_{\lambda} z^{|\lambda|} q^{2 n(\lambda)} u^{\ell(\lambda)} \frac{\left(a ; q^{-1}\right)_{\lambda_{1}}\left(b ; q^{-1}\right)_{\lambda_{1}}}{(q)_{\lambda}} .
\end{aligned}
$$

Let $\nu_{1}, \ldots, \nu_{l}$ denote the distinct parts occurring in $\lambda$, ordered so that $\nu_{1}>$ $\nu_{2}>\cdots>0$, and let $m_{i}$ denote the multiplicity of $\nu_{i}$ in $\lambda$. In terms of these parameters, we have (cf. (2.4)):

$$
\begin{aligned}
\psi_{a b}(u) & =\frac{1}{1-u} \sum_{(m), \nu} \frac{\left(a ; q^{-1}\right)_{\nu_{1}}\left(b ; q^{-1}\right)_{\nu_{1}}}{(q)_{\nu}}\left(z^{\nu_{1}} q^{\nu_{1}\left(\nu_{1}-1\right)} u\right)^{m_{1}} \cdots\left(z^{\nu_{l}} q^{\nu_{l}\left(\nu_{l}-1\right)} u\right)^{m_{l}} \\
& =\frac{1}{1-u} \sum_{\nu} \frac{\left(a ; q^{-1}\right)_{\nu_{1}}\left(b ; q^{-1}\right)_{\nu_{1}}}{(q)_{\nu}}\left\langle z^{\nu_{1}} q^{\nu_{1}\left(\nu_{1}-1\right)} u\right\rangle \cdots\left\langle z^{\nu_{l}} q^{\nu_{l}\left(\nu_{l}-1\right)} u\right\rangle .
\end{aligned}
$$

Each of the terms in this sum, as a rational function of $u$, has a finite set of simple poles. These poles may occur only at the points $u=z^{-r} q^{-r(r-1)}(r=$ $0,1,2, \ldots)$. Therefore, each term is a linear combination of partial fractions of the form $1 /\left(1-u z^{r} q^{r(r-1)}\right)$. Moreover, it is easy to check that the sum of these partial fraction expansions converges coefficientwise (either analytically in a neighborhood of 0 , or in a formal power series ring), so that $\psi_{a b}$ has an expansion of the form

$$
\psi_{a b}(u)=\sum_{r=0}^{\infty} \frac{c_{r}}{1-u z^{r} q^{r(r-1)}}
$$


where $c_{r}$ denotes the (formal) sum of the partial fraction coefficients contributed by the terms of (3.5). By extracting the coefficient of $u^{k}$, note that we therefore have

$$
\sum_{\ell(\lambda) \leq k} z^{|\lambda|} q^{2 n(\lambda)} \frac{\left(a ; q^{-1}\right)_{\lambda_{1}}\left(b ; q^{-1}\right)_{\lambda_{1}}}{(q)_{\lambda}}=\sum_{r=0}^{\infty} z^{k r} q^{k r(r-1)} c_{r} .
$$

Treating $u$ as an analytic variable, the coefficient $c_{0}$ may be computed by means of Lemma 3.3:

$$
c_{0}=\left.\psi_{a b}(u)(1-u)\right|_{u=1}=\frac{(a z)_{\infty}(b z)_{\infty}}{(z)_{\infty}(a b z)_{\infty}} .
$$

Hence, (3.5) implies

$$
\frac{(a z)_{\infty}(b z)_{\infty}}{(z)_{\infty}(a b z)_{\infty}}=\sum_{\nu} \frac{\left(a ; q^{-1}\right)_{\nu_{1}}\left(b ; q^{-1}\right)_{\nu_{1}}}{(q)_{\nu}} \prod_{i}\left\langle z^{\nu_{i}} q^{\nu_{i}\left(\nu_{i}-1\right)}\right\rangle
$$

To compute the general coefficient $c_{r}(r>0)$, we may restrict our attention to those terms in (3.5) that include a pole at $u=z^{-r} q^{-r(r-1)}$, i.e., to those terms with $\nu_{j}=r$ for some $j$. For each such partition $\nu$, we define $\rho_{i}=\nu_{i}-r(1 \leq$ $i<j)$ and $\sigma_{i}=\nu_{i+j}(0 \leq i \leq l-j)$, and observe that the parts of $\sigma$ are bounded by $r$. In terms of these parameters, we may use (3.5) to write the series $c_{r}$ in the form

$$
\begin{aligned}
c_{r}= & \frac{1}{1-z^{-r} q^{-r(r-1)}} \sum_{\rho, \sigma} \frac{\left(a ; q^{-1}\right)_{\rho_{1}+r}\left(b ; q^{-1}\right)_{\rho_{1}+r}}{(q)_{\rho}(q)_{r}}\left[\begin{array}{l}
r \\
\sigma
\end{array}\right] \\
& \cdot \prod_{i}\left\langle z^{\rho_{i}} q^{\left(\rho_{i}+r\right)\left(\rho_{i}+r-1\right)-r(r-1)}\right\rangle \prod_{i}\left\langle z^{\sigma_{i}-r} q^{\sigma_{i}\left(\sigma_{i}-1\right)-r(r-1)}\right\rangle .
\end{aligned}
$$

The $\sigma$-dependence of this series can be eliminated via (2.6), yielding

$$
c_{r}=\frac{\left(a ; q^{-1}\right)_{r}\left(b ; q^{-1}\right)_{r}}{\left(z^{-1} q^{-2(r-1)}\right)_{r}(q)_{r}} \sum_{\rho} \frac{\left(a q^{-r} ; q^{-1}\right)_{\rho_{1}}\left(b q^{-r} ; q^{-1}\right)_{\rho_{1}}}{(q)_{\rho}} \prod_{i}\left\langle\left(z q^{2 r}\right)^{\rho_{i}} q^{\rho_{i}\left(\rho_{i}-1\right)}\right\rangle .
$$

Therefore, if we apply (3.7) (with $a \rightarrow a q^{-r}, b \rightarrow b q^{-r}, z \rightarrow z q^{2 r}$ ), we obtain

$$
\begin{aligned}
& c_{r}=\frac{\left(a ; q^{-1}\right)_{r}\left(b ; q^{-1}\right)_{r}}{\left(z^{-1} q^{-2(r-1)}\right)_{r}(q)_{r}} \frac{\left(a z q^{r}\right)_{\infty}\left(b z q^{r}\right)_{\infty}}{(a b z)_{\infty}\left(z q^{2 r}\right)_{\infty}} \\
& =(-1)^{r} z^{r} q^{3\left(\begin{array}{c}
r \\
2
\end{array}\right)}\left(1-z q^{2 r-1}\right) \frac{\left(a ; q^{-1}\right)_{r}\left(b ; q^{-1}\right)_{r}\left(a z q^{r}\right)_{\infty}\left(b z a^{r}\right)_{\infty}}{(a b z)_{\infty}\left(z q^{r-1}\right)_{\infty}} .
\end{aligned}
$$

In view of (3.6), this completes the proof of (b). A proof of (a) follows similarly.

In $\S 1$, we showed that for special values of the parameter $z$, the series of Corollary 1.4 were "Jacobi-summable", i.e., of the form (0.3) or (0.4), aside 
from multiplicative factors. These special values led to the four generalized identities of Rogers-Ramanujan type in Corollary 1.5. We now consider similar ramifications of Theorem 3.4. That is, we will determine specializations of (as well as certain other operations involving) the parameters $a, b$, and $z$ for which the series of Theorem 3.4 are Jacobi-summable.

As an example of the "other" operations cited above, note that one may regard Theorem 3.4(b) as an identity in the formal power series ring $R[[b]]$, where $R=\mathbf{Z}[[a, z, q]]$. In particular, one may then treat the coefficients of $b^{0}, b^{1}, b^{2}, \ldots$ as formal power series identities in $R$. For each such identity (or $\mathbf{Z}[q]$-linear combination thereof), one may then look for specializations of $a$ and $z$ that are Jacobi-summable. However, one should be aware that, depending on the $\mathbf{Z}[q]$-linear combination involved, the $\lambda$ th term of the infinite series might not be expressible as a quotient of cyclotomic polynomials, and thus not likely to be an interesting $q$-series identity.

Including the four cases already determined in Corollary 1.5, there are a total of 16 distinct families of Rogers-Ramanujan-type identities that can be obtained by some combination of specialization and/or coefficient extraction applied to Theorem 3.4. In some cases, there may be more than one combination of operations that lead to the same identity, but in the list below, we have given only one means of derivation for each identity.

The notation $\left[b^{r}\right]$ will be used as an abbreviation for the coefficient-of- $b^{r}$ operator.

Proposition 3.5. Each of the following operations, when applied to the identities of Theorem 3.4, will produce infinite series that are Jacobi-summable.

(a) Operations to be applied to Theorem 3.4(a):

$$
\begin{array}{ll}
\text { (1) } z=q^{1 / 2}, a=0 ; & \text { (2) } z=q, a=0 ; \\
\text { (3) } z=q, a=-q^{-1 / 2} ; & \text { (4) } z=q,\left(1-\frac{1}{q}\right)\left[a^{1}\right] .
\end{array}
$$

(b) Operations to be applied to Theorem 3.4(b):

(5) $z=q, a=b=0$;

(6) $z=q^{2}, a=b=0$;

(7) $z=q, a=-q^{-1 / 2}, b=0$;

(8) $z=q^{2}, a=-q^{-1 / 2}, b=0$;

(9) $z=q^{2}, a=-q^{-1}, b=0$;

(10) $z=q^{2}, a=-q^{-1 / 2}, b=-q^{-1}$;

(11) $z=q, a=0,\left(1-\frac{1}{q}\right)\left[b^{1}\right]$;

(12) $z=q, a=-1,\left(1-\frac{1}{q}\right)\left[b^{1}\right]$;

(13) $z=q^{2}, a=0,\left[b^{1}\right]+\left(1-\frac{1}{q}\right)\left[b^{1}\right]$;

(14) $z=q^{2}, a=0,\left[b^{1}\right]+\left(1-\frac{1}{q}\right)\left[b^{2}\right]$;

(15) $z=q^{2}, a=-q^{-1 / 2},\left[b^{0}\right]+\left(1-\frac{1}{q}\right)\left[b^{1}\right]$;

(16) $z=q^{2}, a=-q^{-1},\left(1-\frac{1}{q}\right)\left[b^{1}\right]$.

Proof. Consider case (16). By substituting $z=q^{2}, a-q^{-1}$ into Theorem 
3.4(b), we obtain

$$
\begin{aligned}
& \sum_{\ell(\lambda) \leq k} q^{2 n(\lambda)+2|\lambda|-\left(\begin{array}{c}
\lambda_{1}+1 \\
2
\end{array}\right)} \frac{(-q)_{\lambda_{1}}\left(b ; q^{-1}\right) \lambda_{1}}{(q)_{\lambda}} \\
& =\sum_{r=0}^{\infty}(-1)^{r} q^{(2 k+1) r+(2 k+2)\left(\begin{array}{l}
r \\
2
\end{array}\right)} \frac{(-q)_{\infty}\left(b ; q^{-1}\right)_{r}\left(b q^{r+2}\right)_{\infty}\left(1-q^{2 r+1}\right)}{(q)_{\infty}(-b q)_{\infty}},
\end{aligned}
$$

and so the operation $(1-1 / q)\left[b^{1}\right]$ produces the identity

$$
\begin{aligned}
& \sum_{\ell(\lambda) \leq k} q^{2 n(\lambda)+2|\lambda|-\lambda_{1}\left(\lambda_{1}+3\right) / 2} \frac{(-q)_{\lambda_{1}}\left(1-q^{\lambda_{1}}\right)}{(q)_{\lambda}} \\
& =\frac{(-q)_{\infty}}{(q)_{\infty}} \sum_{r=0}^{\infty}(-1)^{r} q^{2 k r+(2 k+2)\left(\begin{array}{l}
r \\
2
\end{array}\right)}\left(1-q^{4 r+2}\right) .
\end{aligned}
$$

Aside from the multiplicative factors, the right side is of the form $(0.4)$, with the identifications $q \rightarrow q^{2 k+2}, a \rightarrow q^{2 k}$, and so we deduce

$$
\sum_{\ell(\lambda) \leq k} q^{2 n(\lambda)+2|\lambda|-\lambda_{1}\left(\lambda_{1}+3\right) / 2} \frac{(-q)_{\lambda_{1}}\left(1-q^{\lambda_{1}}\right)}{(q)_{\lambda}}=\frac{(-q)_{\infty}}{(q)_{\infty}} \prod_{n}\left(1-q^{n}\right),
$$

with the product taken over positive integers $n \equiv 0, \pm 2 \bmod 2 k+2$. The details for the remaining cases are substantially similar and equally easy.

We now review the 16 families of identities produced by the above list. Of course, four of these (case (1), (2), (5), and (6)) appear already in Corollary 1.5, and a fifth (case (13)) reproduces the special case $i=2$ of Andrews' identity (1.12). Another three (cases (7), ${ }^{\prime}(8)$, and (15)) are equivalent to the special cases $i=1,2, k+1$ of the following identity due to Bressoud [B2, (3.8)]:

$$
\sum_{\ell(\lambda) \leq k} q^{2 n_{2}(\lambda)+2\left(\lambda_{i}+\cdots+\lambda_{k}\right)-\lambda_{1}^{2}} \frac{\left(-q ; q^{2}\right)_{\lambda_{1}}}{(q)_{\lambda}}=\frac{\left(-q ; q^{2}\right)_{\infty}}{\left(q^{2} ; q^{2}\right)_{\infty}} \prod\left(1-q^{n}\right),
$$

with the product taken over positive integers $n \equiv 0, \pm(2 i-1) \bmod 4 k+4$.

The remaining eight cases not previously accounted for appear to be new, although it is possible that some of them are analytic counterparts of combinatorial generalizations of the Rogers-Ramanujan identities due to Andrews [A1, A3], and Bressoud [B1, B2]. We list these eight identities below. The notation I $n$ ) is used to refer to the identity corresponding to case $(n)$. In (I3) and (I10) we have substituted $q^{2}$ for $q$ to avoid fractional exponents. In addition to the parameter $n_{2}(\lambda)=\sum \lambda_{i}^{2}$, we will also use

$$
\widehat{n}(\lambda)=n(\lambda)+|\lambda|=\sum_{i}\left(\begin{array}{c}
\lambda_{i}+1 \\
2
\end{array}\right)
$$


as an abbreviation in what follows.

$$
\sum_{\ell(\lambda) \leq k} q^{2 \widehat{n}(\lambda)-\lambda_{1}^{2}} \frac{\left(-q ; q^{2}\right)_{\lambda_{1}}}{\left(q^{2} ; q^{2}\right)_{\lambda}}=\frac{(-q)_{\infty}}{(q)_{\infty}} \prod_{n>0}\left(1-q^{n}\right),
$$$$
n \equiv 0, \pm 1(2 k+2)
$$

(I4)

$$
\sum_{\ell(\lambda) \leq k} q^{\widehat{n}(\lambda)-\lambda_{1}} \frac{\left(1-q^{\lambda_{1}}\right)}{(q)_{\lambda}}=\frac{(-q)_{\infty}}{(q)_{\infty}} \prod_{n>0}\left(1-q^{n}\right)
$$

$$
n \equiv 0, \pm 2(k+2)
$$

$$
\sum_{\ell(\lambda) \leq k} q^{2 \widehat{n}(\lambda)-\left(\begin{array}{c}
\lambda_{1}+1 \\
2
\end{array}\right)} \frac{(-q)_{\lambda_{1}}}{(q)_{\lambda}}=\frac{(-q)_{\infty}}{(q)_{\infty}} \prod_{n>0}\left(1-q^{n}\right)
$$

$$
n \equiv 0, \pm 1(2 k+2)
$$

$$
\begin{aligned}
& \sum_{\ell(\lambda) \leq k} q^{\widehat{4 n}(\lambda)-\lambda_{1}\left(2 \lambda_{1}+1\right)} \frac{(-q)_{2 \lambda_{1}}}{\left(q^{2} ; q^{2}\right)_{\lambda}}=\frac{(-q)_{\infty}}{(q)_{\infty}} \prod_{n>0}\left(1-q^{n}\right) \\
& n \equiv 0, \pm 1(4 k+2) ;
\end{aligned}
$$

$$
\begin{aligned}
& \sum_{\ell(\lambda) \leq k} q^{n_{2}(\lambda)-\lambda_{1}} \frac{\left(1-q^{\lambda_{1}}\right)}{(q)_{\lambda}}=\frac{1}{(q)_{\infty}} \prod_{n>0}\left(1-q^{n}\right), \\
& n \equiv 0, \pm k(2 k+3) ;
\end{aligned}
$$

$$
\begin{aligned}
\sum_{\ell(\lambda) \leq k} q^{n_{2}(\lambda)-\left(\begin{array}{c}
\lambda_{1}+1 \\
2
\end{array}\right) \frac{\left(1-q^{\lambda_{1}}\right)(-q)_{\lambda_{1}-1}}{(q)_{\lambda}}=\frac{(-q)_{\infty}}{(q)_{\infty}} \prod_{n>0}}\left(1-q^{n}\right) \\
n \equiv 0, \pm k(2 k+2) ;
\end{aligned}
$$

$$
\begin{aligned}
& \sum_{\ell(\lambda) \leq k} q^{2 \widehat{n}(\lambda)-2 \lambda_{1}} \frac{\left(1-q^{2 \lambda_{1}}\right)}{(q)_{\lambda}}=\frac{1}{(q)_{\infty}} \prod_{n>0}\left(1-q^{n}\right), \\
& n \equiv 0, \pm 3(2 k+3) \text {; }
\end{aligned}
$$

$$
\begin{aligned}
& \sum_{\ell(\lambda) \leq k} q^{2 \widehat{n}(\lambda)-\lambda_{1}\left(\lambda_{1}+3\right) / 2} \frac{\left(1-q^{\lambda_{1}}\right)(-q)_{\lambda_{1}}}{(q)_{\lambda}}=\frac{(-q)_{\infty}}{(q)_{\infty}} \prod_{n>0}\left(1-q^{n}\right), \\
& n \equiv 0, \pm 2(2 k+2) .
\end{aligned}
$$

The simplest special cases of these identities are obtained by setting $k=1$. In this context, we noted in $\S 1$ that identities (I5) and (I6) are the RogersRamanujan identities. It is also not hard to check that (I11), (I13), and (I14) specialize to the Rogers-Ramanjan identities as well, although in the case of (I14), one must rearrange the terms of the series to verify the coincidence. 
A pair of identities derive from (I7), (I8), and (I15) (or equivalently, from (3.8)) in the case $k=1$; namely,

$$
\begin{aligned}
& \sum_{n=0}^{\infty} q^{n^{2}} \frac{\left(-q ; q^{2}\right)_{n}}{\left(q^{2} ; q^{2}\right)_{n}}=\prod_{n \equiv \pm 1,4(8)} \frac{1}{1-q^{n}}, \\
& \sum_{n=0}^{\infty} q^{n^{2}+2 n} \frac{\left(-q ; q^{2}\right)_{n}}{\left(q^{2} ; q^{2}\right)_{n}}=\prod_{n \equiv \pm 3,4(8)} \frac{1}{1-q^{n}} .
\end{aligned}
$$

It is easy to interpret both of these as combinatorial identities; for example, the series in (3.9) can be viewed as the generating function for pairs of selfconjugate partitions $(\sigma, \tau)$ in which the largest part of $\sigma$ is at most the length of the main diagonal of $\tau$.

Next, recall from $\S 1$ that when $k=1$, identities (I1) and (I2) are wellknown results of Euler. It is also easy to verify that (I4) and (I2) are equivalent in this case. The remaining cases are (I3), (I9), (I10), (I12), and (I16); the corresponding identities are:

$$
\begin{gathered}
\sum_{n=0}^{\infty} q^{n} \frac{\left(-q ; q^{2}\right)_{n}}{\left(q^{2} ; q^{2}\right)_{n}}=\prod_{n \neq 0(4)} \frac{1}{1-q^{n}}, \\
\sum_{n=0}^{\infty} q^{\left(\begin{array}{c}
n+1 \\
2
\end{array}\right) \frac{(-q)_{n}}{(q)_{n}}}=\prod_{n \neq 0(4)} \frac{1}{1-q^{n}}, \\
\sum_{n=0}^{\infty} q^{n} \frac{(-q)_{2 n}}{\left(q^{2} ; q^{2}\right)_{n}}=\left(-q^{3} ; q^{3}\right)_{\infty} \prod_{n \neq 0(6)} \frac{1}{1-q^{n}}, \\
\sum_{n=0}^{\infty} q^{\left(\begin{array}{c}
n+1 \\
2
\end{array}\right)} \frac{(-q)_{n+1}}{(q)_{n}}=\prod_{n=0}^{\infty} \frac{1+q^{2 n+1}}{1-q^{2 n+1}} .
\end{gathered}
$$

Only four identities appear in this list since (I9) and (I12) are equivalent when $k=1$.

We remark that (3.11) is a special case of the $q$-Binomial Identity (take $z \rightarrow q^{1 / 2}$ and $a \rightarrow-q^{1 / 2}$ in (0.2)), (3.12) can be found listed eighth among Slater's catalogue of $130 q$-series identities [Sl], and (3.14) is a special case of a result due to Gauss [A5, Corollary 2.7]. The remaining identity (3.13) does not seem to have explicitly appeared in the literature of $q$-series before.

\section{Plane partitions}

As mentioned in the introduction, we can rederive Proctor's recent plane partition generating functions via Theorem 1.2 in roughly the same way Macdonald proved the MacMahon and Bender-Knuth conjectures via Theorem 1.1. The first step in the derivation of Proctor's identities is to set $q=0$ in Theorem 1.2 and rewrite the result in terms of the root system $C_{n}$.

We may realize $C_{n}$ as the set of vectors $\left\{ \pm 2 e_{i}, \pm e_{i} \pm e_{j}: 1 \leq i<j \leq n\right\}$ and $C_{n}^{+}=\left\{2 e_{i}, e_{i} \pm e_{j}: 1 \leq i<j \leq n\right\}$ as a set of positive roots, where $e_{1}, \ldots, e_{n}$ 
denotes the standard basis of $\mathbf{R}^{n}$. The Weyl group $W_{n}$ of $C_{n}$ is the semidirect product $\mathbf{Z}_{2}^{n} \rtimes S_{n}$, where $S_{n}$ acts by permuting coordinates, and $\mathbf{Z}_{2}^{n}$ acts by changing the signs of coordinates. Note that $\rho=(n, n-1, \ldots, 1)$ is half the sum of $C_{n}^{+}$.

For any $\alpha \in \mathbf{R}^{n}$, let $\Delta_{\alpha}(x)=\Delta_{\alpha}\left(x_{1}, \ldots, x_{n}\right)=\sum_{w \in W_{n}} \operatorname{sgn}(w) x^{w \alpha}$, so that if $\alpha$ is a highest weight vector for $C_{n}$, then $s p_{\alpha}(x)=\Delta_{\alpha+\rho}(x) / \Delta_{\rho}(x)$ is the character of the corresponding representation of $\operatorname{Sp}(2 n, \mathbf{C})$. Note that $\theta=$ $(1, \ldots, 1)$ is the highest weight vector corresponding to the $n$th fundamental weight of $C_{n}$, and recall that $s_{\lambda}$ denotes the Schur function indexed by $\lambda[\mathrm{M}$, I.3].

Theorem 4.1. For any nonnegative integer $k$, we have

$$
x^{k \theta} s p_{k \theta}\left(x_{1}, \ldots, x_{n}\right)=\sum_{\lambda_{1} \leq k} s_{2 \lambda}\left(x_{1}, \ldots, x_{n}\right) .
$$

Proof. From the definition of $\Psi$ in $\S 1$, we have

$$
\Psi(x ; 0)=\prod_{\alpha \in A_{n-1}^{+}}\left(1-x^{\alpha}\right) \prod_{\alpha \in C_{n}^{+}}\left(1-x^{\alpha}\right)^{-1},
$$

where $A_{n-1}^{+}=\left\{e_{i}-e_{j}: 1 \leq i<j \leq n\right\}$ denotes a set of positive roots for the subsystem $A_{n-1}$. Using the Weyl denominator formulas for $A_{n-1}$ and $C_{n}$, it follows that

$$
\Psi(x ; 0)=x^{\rho_{A}-\rho} \frac{\prod_{\alpha \in A_{n-1}^{+}}\left(x^{-\alpha / 2}-x^{\alpha / 2}\right)}{\prod_{\alpha \in C_{n}^{+}}\left(x^{-\alpha / 2}-x^{\alpha / 2}\right)}=\frac{x^{\rho_{A}-\rho}}{\Delta_{\rho}\left(x^{-1}\right)} \sum_{w \in S_{n}} \operatorname{sgn}(w) x^{-w \rho_{A}},
$$

where $\rho_{A}$ denotes half the sum of $A_{n-1}^{+}$. Since $\rho_{A}-\rho=-1 / 2(n+1) \theta$ is $S_{n}$-invariant, it follows that

$$
\Psi(x ; 0)=\Delta_{\rho}^{-1}\left(x^{-1}\right) \sum_{w \in S_{n}} \operatorname{sgn}(w) x^{-w \rho} .
$$

Substituting $q=0$ into Theorem 1.2, we obtain

$$
\sum_{k \geq 0} u^{k} \sum_{\lambda_{1} \leq k} s_{2 \lambda}\left(x_{1}, \ldots, x_{n}\right)=\sum_{s \in \mathbf{Z}_{2}^{n}} \Psi(s x ; 0) \frac{1}{1-u x^{\theta-s \theta}} .
$$

Therefore, since $w \Delta_{\rho}=\operatorname{sgn}(w) \Delta_{\rho}$ for all $w \in W_{n}$, an application of (4.1) yields

$\sum_{\lambda_{1} \leq k} s_{2 \lambda}\left(x_{1}, \ldots, x_{n}\right)=\frac{1}{\Delta_{\rho}\left(x^{-1}\right)} \sum_{s \in \mathbf{Z}_{2}^{n}} x^{k \theta} \sum_{w \in S_{n}} \operatorname{sgn}(s w) x^{-s w(k \theta+\rho)}=x^{k \theta} s p_{k \theta}\left(x^{-1}\right)$.

Since the characters of $S p(2 n, \mathbf{C})$ are self-dual $\left(s p_{k \theta}(x)=s p_{k \theta}\left(x^{-1}\right)\right)$, the result follows.

We remark that this result is essentially equivalent to the "CY" case of Theorem 2 in [P2], the only difference being that Proctor expressed the character 
$s p_{k \theta}$ as a quotient of determinants. However, the proofs are substantially different; Proctor's proof relies on a construction of representations of the symplectic group due to DeConcini, Lakshmibai, and Seshadri [D]. We also note that the proof given here need not involve the theory of Hall-Littlewood functions (via Theorem 1.2), since the partial fraction method could also have been employed after setting $q=0$.

There are two ways to specialize the variables $x_{i}$ so that the character $s p_{k \theta}$ can be factored in closed form. We have included details of the proof for the sake of completeness, even though this calculation can also be found in [P2] (cf. also Examples I.5.17, 19 in [M]).

\section{Proposition 4.2.}

$$
\begin{aligned}
& s p_{k \theta}\left(q^{n}, q^{n-1}, \ldots, q\right) \\
& =q^{-k\left(\begin{array}{c}
n+1 \\
2
\end{array}\right)} \prod_{1 \leq i \leq n} \frac{1-q^{2 k+2 i}}{1-q^{2 i}} \prod_{1 \leq i<j \leq n} \frac{1-q^{2 k+i+j}}{1-q^{i+j}} . \\
& s p_{k \theta}\left(q^{2 n-1}, q^{2 n-3}, \ldots, q\right) \\
& =q^{-k n^{2}} \prod_{1 \leq i \leq n} \frac{1-q^{2 k+2 i}}{1-q^{2 i}} \prod_{1 \leq i<j \leq n} \frac{1-q^{4 k+2 i+2 j}}{1-q^{2 i+2 j}} .
\end{aligned}
$$

Proof. For any $\beta \in \mathbf{R}^{n}$, consider the effect of the substitution $x_{i} \rightarrow q^{\beta_{i}}$ in $\Delta_{k \theta+\rho}$ :

$$
\Delta_{k \theta+\rho}(x) \rightarrow \sum_{w \in W_{n}} \operatorname{sgn}(w) q^{\langle\beta, w(k \theta+\rho)\rangle}=\sum_{w \in W_{n}} \operatorname{sgn}(w) q^{\langle w \beta, k \theta+\rho\rangle},
$$

where $\langle$,$\rangle denotes the standard Euclidean inner product. It follows that$ if $\beta=\rho$, then $\Delta_{k \theta+\rho}$ becomes the Weyl denominator $\Delta_{\rho}$ in the variables $x_{i}=q^{k+\rho_{i}}=q^{k+n-i+1}$. Hence,

$$
\begin{aligned}
s p_{k \theta}\left(q^{n}, \ldots, q\right) & =\frac{\Delta_{\rho}\left(q^{k+n}, \ldots, q^{k+1}\right)}{\Delta_{\rho}\left(q^{n}, \ldots, q^{1}\right)} \\
& =\prod_{i} \frac{q^{k+i}-q^{-(k+i)}}{q^{i}-q^{-i}} \prod_{i<j} \frac{q^{k+(i+j) / 2}-q^{-k-(i+j) / 2}}{q^{(i+j) / 2}-q^{-(i+j) / 2}},
\end{aligned}
$$

from which (a) follows.

To prove (b), note that $W_{n}$ is also the Weyl group of the root system $B_{n}$, so if we choose $\beta=(2 n-1,2 n-3, \ldots, 1)$ (i.e., the sum of $B_{n}^{+}$in the standard coordinates), then (4.2) will imply that $\Delta_{k \theta+\rho}$ becomes the Weyl denominator 
$\Delta^{\prime}$ for $B_{n}$ in the variables $x_{i}=q^{2 k+2 \rho_{i}}=q^{2 k+2 n-2 i+2}$. We therefore have

$$
\begin{gathered}
s p_{k \theta}\left(q^{2 n-1}, q^{2 n-3}, \ldots, q\right)=\frac{\Delta^{\prime}\left(q^{2 k+2 n}, \ldots, q^{2 k+2}\right)}{\Delta^{\prime}\left(q^{2 n-1}, \ldots, q^{1}\right)} \\
=\prod_{i} \frac{q^{k+i}-q^{-(k+i)}}{q^{i}-q^{-i}} \prod_{i<j} \frac{q^{2 k+i+j}-q^{-(2 k+i+j)}}{q^{i+j}-q^{-(i+j)}}
\end{gathered}
$$

from which (b) follows.

Finally, we consider the combinatorial consequences of Theorem 4.1 and Proposition 4.2. Recal that a plane partition of $r$ is a matrix-style array $P=$ $\left(p_{i j}\right)_{i, j \geq 1}$ of nonnegative integers with weakly decreasing rows and columns (i.e., $p_{i, j} \geq p_{i, j+1}, p_{i, j} \geq p_{i+1, j}$ ) such that $\sum p_{i j}=r$. A shifted plane partition $S=\left(s_{i j}\right)$ is defined similarly, except that the entries are restricted to a domain on or above the main diagonal (i.e., $s_{i j}$ is defined only for $1 \leq i \leq j$ ). Let $\mathscr{P}_{m, n}$ denote the set of plane partitions whose nonzero entries are bounded by $m$ and are restricted to lie in the first $n$ rows and columns, and let $\mathscr{S}_{m, n}$ denote the corresponding set of shifted plane partitions (so that $s_{i j} \leq m$, and $s_{i j} \neq 0$ only if $\left.1 \leq i \leq j \leq n\right)$.

Proctor's pair of plane partition generating functions (see the "CYI" and "CYH" cases in Theorem 1 of [P2]) are the following.

\section{Corollary 4.3.}

$$
\prod_{1 \leq i \leq n} \frac{1-q^{2 k+2 i}}{1-q^{2 i}} \prod_{1 \leq i<j \leq n} \frac{1-q^{2 k+i+j}}{1-q^{i+j}}=\sum_{r=0}^{\infty} s_{2 k, n}(r) q^{r},
$$

where $s_{2 k, n}(r)$ is the number of shifted plane partitions of $r$ in $\mathscr{S}_{2 k, n}$ with even entries on the main diagonal.

$$
\prod_{1 \leq i \leq n} \frac{1-q^{2 k+2 i}}{1-q^{2 i}} \prod_{1 \leq i<j \leq n} \frac{1-q^{4 k+2 i+2 j}}{1-q^{2 i+2 j}}=\sum_{r=0}^{\infty} p_{2 k, n}(r) q^{r},
$$

where $p_{2 k, n}(r)$ is the number of symmetric (i.e., $p_{i j}=p_{j i}$ ) plane partitions of $r$ in $\mathscr{P}_{2 k, n}$ with even entries on the main diagonal.

Proof. By Theorem 4.1 and Proposition 4.2(a), we have

$$
\prod_{i} \frac{1-q^{2 k+2 i}}{1-q^{2 i}} \prod_{i<j} \frac{1-q^{2 k+i+j}}{1-q^{i+j}}=\sum_{\lambda_{1} \leq k} s_{2 \lambda}\left(q^{n}, \ldots, q\right) .
$$

The right side may be interpreted as the generating function for column-strict (i.e., $p_{i, j}>p_{i+1, j}$ ) plane partitions $P$ that (1) fit inside an $n \times 2 k$ rectangle, (2) have entries bounded by $n$, and (3) have an even number of nonzero entries in each row. (This is an immediate consequence of the well-known combinatorial interpretation of Schur functions; see [M, I.5], for example.) Corresponding to $P$, let us associate a shifted plane partition $S=\left(s_{i j}\right)$ defined so that $s_{i j}$ is the number of entries in the $i$ th row of $P$ that are at least $j-i+1$. Note that for 
each $i$, the $i$ th rows of $P$ and $S$ are conjugate linear partitions, so $P$ and $S$ have the same sum-of-entries. The diagonal $s_{i i}$ is the number of nonzero entries in the $i$ th row of $P$ (and therefore even), and the largest entry $s_{11}$ is clearly at most $2 k$. Since the entries of $P$ are at most $n$, it also follows that the first row of $S$ has at most $n$ nonzero entries, and thus, $S \in \mathscr{S}_{2 k, n}$. It is easy to check that the map $P \mapsto S$ is a bijection, and thus (a) follows.

For (b), we apply Theorem 4.1 and Proposition 4.2(b) to obtain

$$
\prod_{i} \frac{1-q^{2 k+2 i}}{1-q^{2 i}} \prod_{i<j} \frac{1-q^{4 k+2 i+2 j}}{1-q^{2 i+2 j}}=\sum_{\lambda_{1} \leq k} s_{2 \lambda}\left(q^{2 n-1}, q^{2 n-3}, \ldots, q\right) .
$$

Interpret the right side as the generating function for column-strict plane partitions $P$ as above, except that in place of condition (2), we instead require that the positive entries be odd integers of size at most $2 n-1$. If we associate with $P$ the shifted plane partition $S=\left(s_{i j}\right)$ obtained by defining $s_{i j}$ as the number of entries in the $i$ th row of $P$ that are at least $2 j-2 i+1$, then $P \mapsto S$ is still a bijection, but no longer preserves the sum-of-entries; an odd entry $2 m-1$ in $P$ will contribute $m$ to the weight of $S$. Since the sum-of-entries on the main diagonal of $S$ is the number of positive entries in $P$, it follows that if we extend $S$ to a symmetric plane partition $S^{\prime}$ by defining $s_{i j}^{\prime}=s_{j i}^{\prime}=s_{i j}$ for $i \leq j$, then the bijection $P \mapsto S^{\prime}$ will preserve weight, and thus (b) follows.

Additional notes. I would like to thank the referee for pointing out that most of the results in $\S 4$ were also proved by J. Désarménien in the Proceedings of the 15th Séminaire Lotharingien de Combinatoire (1987). To be precise, Theorem 4.1 and the pair of generating functions (4.3) and (4.4) were also proved by Désarménien, and in essentially the same way as the proofs given here. However, the particular interpretations of (4.3) and (4.4) as generating functions of shifted or symmetric plane partitions (Corollary 4.3), rather than the more obvious column-strict interpretations, are due to Proctor. The special cases of (4.3) and (4.4) corresponding to $q=1$ were also proved by DesainteCatherine and Viennot (Lecture Notes in Math., vol. 1234, Springer, New York).

It is also interesting to note that identity (2.2) first appeared in a paper by Philip Hall (Comment. Math. Helv. 11 (1938), 126-129), in connection with the enumeration of automorphisms of abelian $p$-groups.

\section{REFERENCES}

[A1] G. E. Andrews, Partition theorems related to the Rogers-Ramanujan identities, J. Combin. Theory 2 (1967), 422-430.

[A2] _ - An analytic generalization of the Rogers-Ramanujan identities for odd moduli, Proc. Nat. Acad. Sci. U.S.A. 71 (1974), 4082-4085.

[A3] _- On the general Rogers-Ramanujan theorem, Mem. Amer. Math. Soc. No. 152 (1974).

[A4] _ Problems and prospects for basic hypergeometric functions, The Theory and Application of Special Functions (R. Askey, ed.), Academic Press, New York, 1975, pp. 191-224. 
[A5] _ The theory of partitions, Encyclopedia of Mathematics and its Applications, vol. 2, Addison-Wesley, Reading, Mass., 1976.

[A6] _ Partitions and Durfee dissection, Amer. J. Math. 101 (1979), 735-742.

[A7] _-, Plane partitions (I): The MacMahon conjecture, Studies in Foundations and Combinatorics, Adv. in Math. Suppl. Stud., vol. 1, Academic Press, New York, 1978, pp. 131-150.

[A8] _ Plane partitions (II): The equivalence of the Bender-Knuth and MacMahon conjectures, Pacific J. Math. 72 (1977), 283-291.

[A9] _ $\quad$ - q-series: their development and application in analysis, combinatorics, physics, and computer algebra, CBMS Regional Conf. Ser. in Math., no. 66, Amer. Math. Soc., Providence, R.I., 1986.

[B1] D. M. Bressoud, A generalization of the Rogers-Ramanujan identities for all moduli, J. Combin. Theory Ser. A 27 (1979), 64-68.

[B2] _ Analytic and combinatorial generalizations of the Rogers-Ramanujan identities, Mem. Amer. Math. Soc. No. 227 (1980).

[B3] __ Lattice paths and the Rogers-Ramanujan identities, Pennsylvania State Univ. preprint.

[D] C. DeConcini, Symplectic standard tableaux, Adv. in Math. 34 (1979), 1-27.

[G1] B. Gordon, A combinatorial generalization of the Rogers-Ramanujan identities, Amer. J. Math. 83 (1961), 393-399.

[G2] _ A proof of the Bender-Knuth conjecture, Pacific J. Math. 108 (1983), 99-113.

[Go] I. P. Goulden, Exact values for degree sums over strips of Young diagrams, Univ. of Waterloo preprint.

[M] I. G. Macdonald, Symmetric functions and Hall polynomials, Oxford, 1979.

[P1] R. A. Proctor, Bruhat lattices, plane partition generating functions, and minuscule representations, European J. Combin. 5 (1984), 331-350.

[P2] - New symmetric plane partition identities from invariant theory work of DeConcini and Procesi, European J. Combin. (to appear).

[SS] B. E. Sagan and R. P. Stanley, Robinson-Schensted algorithms for skew tableaux, J. Combin. Theory Ser. A (to appear).

[Sl] L. J. Slater, Further identities of the Rogers-Ramanujan type, Proc. London Math. Soc. (2) 54 (1952), 147-167.

[St] R. P. Stanley, Symmetries of plane partitions, J. Combin. Theory Ser. A 43 (1986), 103-113.

[SZ] D. Stanton and D. Zeilberger, The Odlyzko conjecture and O'Hara's unimodality proof, Proc. Amer. Math. Soc. 107 (1989), 39-42.

Department of Mathematics, University of Michigan, Ann Arbor, Michigan 481091003 